University of South Florida

DIGITAL COMMONS

Digital Commons @ University of

@ UNIVERSITY OF SOUTH FLORIDA

South Florida

$5-9-2008$

\title{
A Nested Model Study of the Loop Current Generated Variability and Its Impact on the West Florida Shelf
}

\author{
Alexander Barth \\ University of Liège \\ Aida Alvera-Azcarate \\ University of Liège \\ Robert H. Weisberg \\ University of South Florida, weisberg@usf.edu
}

Follow this and additional works at: https://digitalcommons.usf.edu/msc_facpub

Part of the Marine Biology Commons

\section{Scholar Commons Citation}

Barth, Alexander; Alvera-Azcarate, Aida; and Weisberg, Robert H., "A Nested Model Study of the Loop Current Generated Variability and Its Impact on the West Florida Shelf" (2008). Marine Science Faculty Publications. 135.

https://digitalcommons.usf.edu/msc_facpub/135

This Article is brought to you for free and open access by the College of Marine Science at Digital Commons @ University of South Florida. It has been accepted for inclusion in Marine Science Faculty Publications by an authorized administrator of Digital Commons @ University of South Florida. For more information, please contact digitalcommons@usf.edu. 


\title{
A nested model study of the Loop Current generated variability and its impact on the West Florida Shelf
}

\author{
A. Barth, ${ }^{1,2}$ A. Alvera-Azcárate, ${ }^{1,2}$ and R. H. Weisberg ${ }^{1}$ \\ Received 7 August 2007; revised 28 November 2007; accepted 7 February 2008; published 9 May 2008.
}

[1] A West Florida Shelf model based on the Regional Ocean Modeling System (ROMS)

is nested in the North Atlantic Hybrid Coordinate Ocean Model (NAT HYCOM). The focus of this work is the study of the impact of the Loop Current on the West Florida Shelf. In order to assess the model's accuracy, it is compared quantitatively to in situ temperature and velocity measurements on the shelf. A series of sensitivity experiments are conducted to determine the appropriate wind forcing, sea surface temperature relaxation, and mixing scheme. By the inclusion of the Loop Current, we are able to study the propagation of an anticyclonic vortex detaching from the Loop Current. We found that the ambient gradient of potential vorticity is able to explain the vortex path and speed. The statistics of such Loop Current generated flow features were examined by including a tracer marking Loop Current water. This allows to track the Loop Current water on the West Florida Shelf and to quantify the amount of Loop Current water reaching the shelf.

Citation: Barth, A., A. Alvera-Azcárate, and R. H. Weisberg (2008), A nested model study of the Loop Current generated variability and its impact on the West Florida Shelf, J. Geophys. Res., 113, C05009, doi:10.1029/2007JC004492.

\section{Introduction}

[2] The generally wide West Florida Shelf (WFS) extends about $200 \mathrm{~km}$ to the shelf break followed by a steep shelf slope (Figure 1). The shelf circulation is mainly driven by winds and atmospheric heat fluxes [He and Weisberg, 2003b], but the circulation may also be impacted by the adjoint boundary current, the Gulf of Mexico Loop Current (LC). Northerly (southerly) winds produce upwelling (downwelling) along the west Florida coast because of the orientation of the coast line [Weisberg et al., 2000]. Since the surface heat fluxes control the stratification, the Ekman dynamics are also influenced by the atmospheric heat input [e.g., Weisberg et al., 2001]. Accurate local forcing is therefore a fundamental ingredient for modeling the WFS. Beyond the shelf break, the variability is dominated by the LC. The LC is a branch of the western boundary current entering the Gulf of Mexico through the Yucatan Channel and it describes an anticyclonic loop before exiting the Gulf of Mexico through the Florida Straits. Instabilities modulate the path of this current. Occasionally, the LC contacts shallow isobaths near the Dry Tortugas, generating topographic Rossby waves that travel northward along the shelf break [Hetland et al., 1999]. Through this mechanism, and in combination with local forcing, deep water can be advected onto the shelf through the bottom Ekman layer [He and Weisberg, 2003a; Weisberg and He, 2003]. A

\footnotetext{
${ }^{1}$ College of Marine Science, University of South Florida, St. Petersburg, Florida, USA.

${ }^{2}$ Now at Department of Astrophysics Geophysics and Oceanography, University of Liege, Liege, Belgium.
}

Copyright 2008 by the American Geophysical Union. 0148-0227/08/2007JC004492 general review of the WFS circulation is provided by Weisberg et al. [2005].

[3] Previous numerical model studies of the WFS included only an idealized barotropic LC [He and Weisberg, 2003b; Weisberg and He, 2003]. The impact of a realistic LC on the shelf dynamics in terms of across-shelf water mass and momentum fluxes, the extent of the LC influence on the shelf, and its predictability remain unclear. Our nesting strategy is a first step in addressing such questions in the context of a baroclinic model of the WFS based on the Regional Ocean Modeling System (ROMS) [Shchepetkin and McWilliams, 2005]. It is driven by open boundary conditions from the GODAE North Atlantic Hybrid Coordinate Ocean Model (NAT HYCOM) [Chassignet et al., 2007] while also being forced locally through wind stress and buoyancy fluxes.

[4] Before a model can be used to answer such scientific questions, its validity needs to be tested using various observations. We compare this nested model against in situ temperature at various depths from several stations and ADCP currents measurements.

[5] The validation exercise will provide us an error estimate of the corresponding model variable. It is useful to compare the model error against the error of a reference system [Murphy, 1988]. Often persistence or climatology is used as a reference to establish the improvement or degradation relative to this baseline. For short-term integrations, usually a model skill is assessed relative to persistence [e.g., Demirov et al., 2003] while for long-term integrations, climatology represents a more useful baseline [e.g., Kara and Hurlburt, 2006; Barron et al., 2006]. Here, the outer model, which provides the boundary condition for the nested model, is used as a reference to assess the skill of 


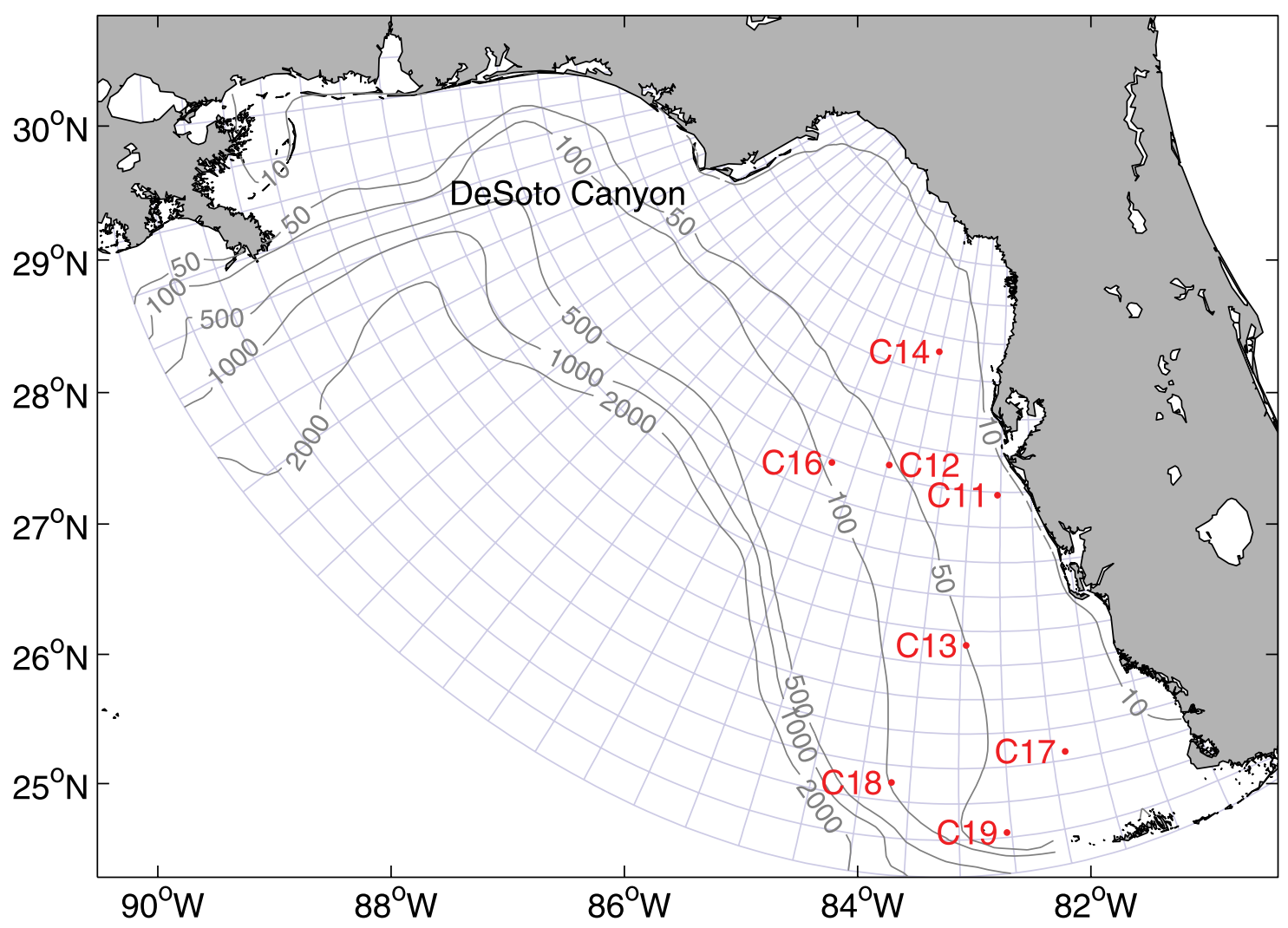

Figure 1. Model domain and location of the in situ stations. The contours represent the model bathymetry. Every fourth grid line is also shown.

a 1-year hindcast experiment. Since the outer model assimilates sea surface temperature (SST) and sea surface height (SSH) [Chassignet et al., 2007] it represents arguably a stricter reference system for model verification and skill assessment than climatology.

[6] However, the objective of this model verification is not to compare the accuracy ROMS and HYCOM in general terms, since both models implementations, in particular the domain and resolution, are too different. Since the WFS ROMS model is nested in NAT HYCOM and has a higher resolution, it is actually expected that the nested model performs better than the outer model which is after all the main reason why models are nested. However, the nested model does not have to be necessarily better than the outer model in all circumstances, since the model nesting can introduce spurious variability at the model boundary which can affect the accuracy inside of the domain. This spurious variability is related to the fact that the inviscid hydrostatic primitive equations with open boundaries is an ill-posed problem [Oliger and Sundstrom, 1978; Browning et al., 1990]. Also the nested grid model can contain smallscale processes which are unable to propagate out of the nested domain. In the present nested model configuration, the WFS ROMS open boundary intersects the LC, which is a strong and highly variable current. The verification of the nested model relative to the outer model allows us to establish if a model nesting can be successful in this case, i.e., if the benefit of the increased resolution outweighs the problems associated with the open boundary.

[7] An integral part of the model verification is the model calibration, since both steps are part of an iterative process in the model development. For the clarity and simplicity, we will first present the best configuration of the model and justify a posteriori the configuration by conducting a sensitivity study. This sensitivity analysis includes (1) the choice of the wind forcing, (2) the SST relaxation, (3) the mixing scheme and vertical resolution and (4) horizontal resolution and bathymetry. The choice of these three factors is guided by the fact that the currents and temperature on the shelf are essentially locally forced. Therefore, we will focus our attention on heat and momentum fluxes at the surface and how they are distributed vertically.

[8] The model validation and calibration use in situ temperature and velocity observations. The distribution of the moorings on the West Florida Shelf (Figure 1) evolved over time to address several issues. First, we were interested in observing the fully three-dimensional structure of the inner shelf, particularly offshore of the region between Tampa Bay and Charlotte Harbor know to be a red tide epicenter. Second, the paucity of available surface wind data for forcing regional ocean circulation models necessitated measurements distributed over the west Florida shelf. In combination these two factors resulted in moorings being placed on the $10-\mathrm{m}, 20-\mathrm{m}$, and $25-\mathrm{m}$ isobaths offshore of Sarasota, FL and moorings being placed on the $50-\mathrm{m}$ isobath offshore of Tampa Bay and Charlotte Harbor, with ADCPs on all and surface meteorological instruments on the $25-\mathrm{m}$ and $50-\mathrm{m}$-isobath moorings. We then added moorings on the $75-\mathrm{m}$ isobath offshore of Tampa Bay to look at flows at the shelf break, the 20-m isobath offshore of Pasco Co. in a cooperative arrangement to satisfy Pasco Co. needs as well as to bolster the requirements given above. 
We then put additional attention to the region farther south, adding a mooring on the $25-\mathrm{m}$ isobath just NE of the Florida Keys to consider flow paths owing to the geometry of the keys, and for these same reasons we added moorings at the cut between Rebecca Shoals and the Dry Tortugas and at the shelf break just northwest of the Dry Tortugas to look at pathways for flow egress and ingress to the WFS and interaction with the Loop Current/Florida Current. The mooring on the shelf NE of the Dry Tortugas also added to the surface meteorological measurements. In summary, these moorings continue to provide information on the inner shelf circulation that have been of use in describing the responses of the WFS circulation to synoptic, seasonal, and interannual forcing [e.g., Weisberg et al., 2005], demonstrating that the inner shelf circulation is fully three-dimensional even into very shallow water, and helping to define the structure of the inner shelf in terms of the momentum balances that apply [e.g., Li and Weisberg, 1999; Weisberg et al., 2001; Liu and Weisberg, 2007].

[9] Previous observation and model studies [Weisberg and He, 2003; Walsh et al., 2003] show that eddies and filaments from the LC play an important role, together with local wind forcings, in the advection of nutrients on the shelf. In the present paper, the propagation mechanism of a LC vortex observed in altimetry and reproduced by the model is studied. Intriguingly, this vortex propagates in the opposite direction of the background flow. Although isolated studies of such events exist, little is known about their frequency and their total contribution to the water properties on the WFS. We will try to address this question using a tracer experiment which shows the transport and dilution of LC water reaching the shelf.

[10] HYCOM (as a continuation of the MICOM) is a well tested and validated model for the basin scale. It is used for the Atlantic Ocean [e.g., Chassignet et al., 2007], Indian Ocean [e.g., Srinivasan et al., 2007; Bertino et al., 2007], Pacific [e.g., Kara et al., 2008] and now also for the Global Ocean [Smedstad et al., 2007]. Its isopycnal coordinate makes it well suited to preserve water mass properties even over a long-term integration. Only recently, HYCOM is also implemented for coastal and regional application [e.g., Halliwell, 2004; DeRada et al., 2006; Kourafalou et al., 2006]. ROMS on the other hand is traditionally applied in regional configuration [e.g., Marchesiello et al., 2001; Wilkin et al., 2005; Di Lorenzo et al., 2005]. Its terrain following $s$ coordinate and its modules (e.g., sediment transport model, wave model) make this model a good choice for regional and small-scale application. By using the output of a HYCOM for the large-scale to drive a regional model of the West Florida Shelf we try to combine in a nesting system the advantages from both models.

[11] In section 2, the model and its WFS implementation are described. Model results are compared to observations in section 3 and the model skill is assessed. The model is calibrated in section 4 by determining the sensitivity of its skill to different wind forcings, different strengths of SST relaxation, mixing schemes, and different vertical and horizontal resolutions. After the model is validated and calibrated, flow features generated by the LC are studied. In section 5, the propagation of an isolated anticyclonic vortex of LC water is examined. The following section assess the statistics of such events by adding a tracer to the model simulation indicating the presence of LC water. We finish with the conclusions and summarize the findings in section 7 .

\section{Model Implementation}

[12] The WFS model is based on ROMS [Shchepetkin and McWilliams, 2005], a hydrostatic, 3D, primitive equation, free-surface model using an $s$ coordinate in the vertical. The horizontal curvilinear grid resolution varies from $4 \mathrm{~km}$ near the coast to $10 \mathrm{~km}$ at the boundary which matches approximately the resolution of the outer model. Studies such as that by Spall and Holland [1991] have shown that an abrupt change in resolution leads to errors at the model boundary. To the south, the domain extends to the Florida Keys. The western extent has been chosen such that the Mississippi River water can be included directly in the model. Observations show that Mississippi River water can flow along the West Florida shelf [Hu et al., 2005; Weisberg et al., 2005]. To the southwest, the domain includes a large enough fraction of the LC that eddies and filaments can be generated inside the model domain since these processes are also known to affect the WFS.

[13] In the WFS ROMS model, the depth $z$ is transformed into a terrain following $s$ coordinate [Song and Haidvogel, 1994; Shchepetkin and McWilliams, 2005] defined by

$$
z(x, y, \sigma)=\sigma h_{\min }+C(\sigma)\left(h(x, y)-h_{\min }\right)
$$

where $h(x, y)$ is the depth, $\sigma$ is the vertical distance from the surface measured as a fraction of the local water column thickness, and $C(\sigma)$, a function that defines the vertical grid spacing, is given by

$$
C(\sigma)=\left(1-\theta_{b}\right) \frac{\sinh (\theta \sigma)}{\sinh (\theta)}+\frac{1}{2} \theta_{b}\left(\frac{\tanh (\theta(\sigma+1 / 2))}{\tanh (\theta / 2)}-1\right),
$$

with the following parameters $\theta=5, \theta_{b}=0.4$ and $h_{\min }$ $=50 \mathrm{~m}$. The vertical grid of the WFS ROMS model contains 32 levels with regular discretization of the variable $\sigma$. This $s$ coordinate is similar to a $\sigma$ coordinate in deep water but in swallow water the resolution is more evenly distributed in the vertical.

[14] To implement a nested model, it is generally preferable to use forcing fields as close as possible to the outer model in order to avoid inconsistencies. We departed from this basic rule only if we could improve the WFS model results. Like the NAT HYCOM, the WFS model surface heat flux is forced by NOGAPS (Navy Operational Global Atmospheric Prediction System) variables, in particular, air temperature, relative humidity, cloud fraction and shortwave radiation. The other heat flux components (latent and sensible heat flux and long-wave radiation) are computed by the WFS ROMS model internally using bulk formulae [Fairall et al., 1996]. Initially, we also used NOGAPS winds, but a significant improvement was obtained by optimal interpolated (OI) winds combining NCEP NAM winds (National Centers for Environmental Prediction, North American Mesoscale Model) with in situ wind measurements (see He et al. [2004] and also section 4.2). We continue to use NOGAPS for the thermal 
forcings in order to have a buoyancy flux consistent with the outer model.

[15] We also obtain more realistic temperatures by relaxing the model SST to a cloud-free optimal interpolated SST based on AVHRR (Advanced Very High Resolution Radiometer), GOES (Geostationary Operational Environmental Satellites), MODIS (MODerate Resolution Imaging Spectroradiometer) and TMI (TRMM Microwave Imager), as described by He et al. [2003]. The heat flux correction has the following form [Barnier et al., 1995]:

$$
Q_{c}=\alpha\left(T(z=0)-T^{o}\right)
$$

where $T^{o}$ is the observed SST and $T(z=0)$ is the model surface temperature and the coefficient $\alpha=-47 \mathrm{Wm}^{-2} \mathrm{~K}^{-1}$. The sensitivity of the model solution with regards to this model parameter is examined in section 4.1. The model is also forced by river inflow. The climatological river runoffs from US Geological Survey for the Mississippi, Mobile, Apalachicola, Suwannee, Hillsborough, Caloosahatchee and Shark Rivers are used.

[16] The WFS ROMS model implementation uses the Mellor-Yamada 2.5 turbulence scheme [Mellor and Yamada, 1982]. The horizontal pressure gradient is computed using the spline density Jacobian formulation by Shchepetkin and McWilliams [2003].

\subsection{Bathymetry}

[17] At the nesting boundary, it is critical that outer and nested models have a consistent bathymetry in order to avoid systematic discrepancies in the transport crossing the nested model domain. In the interior of the domain, the WFS ROMS bathymetry gradually transitions to an ETOPO5 bathymetry. On the WFS, the ROMS bathymetry is more representative because of the increased horizontal resolution and because it has a minimum depth of $2 \mathrm{~m}$.

\subsection{Large-Scale Model}

[18] The outer model used for the nesting is the North Atlantic implementation of HYCOM run by the Naval Research Laboratory, MS, USA. HYCOM is a hybrid coordinate ocean model. In stratified, deep ocean water it uses an isopycnal coordinate, while near the surface and in shallow water it uses a $z$ or $\sigma$ coordinate. The turbulence closure of the NAT HYCOM implementation is the K-Profile Parametrization of Large et al. [1994]. NAT HYCOM uses the atmospheric forcing fields produced by NOGAPS and its surface temperature is relaxed toward MODAS SST: for a $20 \mathrm{~m}$ deep mixed layer, NAT HYCOM will converge toward MODAS SST after 30 days [Chassignet et al., 2007]. HYCOM also assimilates SSH using the method of Cooper and Haines [1996]. The HYCOM fields are available by FTP, OPeNDAP or LAS at http://www.hycom.org/dataserver/.

\subsection{Initialization}

[19] The WFS model is initialized on the 1 January 2004 from the linearly interpolated temperature, salinity, horizontal velocity and surface elevation NAT HYCOM fields. At places where the WFS ROMS bathymetry is deeper than the NAT HYCOM bathymetry, the bottom value of NAT HYCOM is repeated vertically. Since the NAT HYCOM and WFS ROMS minimum depths are different, some coastal points in NAT HYCOM are land points while they are sea grid points in WFS ROMS. In these cases the temperature and salinity are horizontally extrapolated. However, the velocity at those points is initialized with $0 \mathrm{~m} \mathrm{~s}^{-1}$ since a land point in the outer model corresponds to a vanishing normal velocity boundary condition.

\subsection{Nesting Procedure}

[20] The open boundary conditions are interpolated in the same way as the initial conditions explained previously. For the internal velocity, temperature and salinity, we used radiative boundary conditions [Marchesiello et al., 2001] with a nudging term acting at the boundary and over a transition zone (flow relaxation scheme). This relaxes the ROMS model solution toward the NAT HYCOM fields over this transition zone. For instance, the temperature equation includes a term like

$$
\frac{\partial T}{\partial t}=\ldots+c\left(T-T_{\mathrm{OCGM}}\right)
$$

where $T$ and $T_{\mathrm{OCGM}}$ are the WFS ROMS model and the ocean general circulation model (here NAT HYCOM) temperatures. The coefficient $c$ is the strength of the flow relaxation. If $j$ is the grid index perpendicular to the nesting boundary with $j=1$ at the open boundary, then $c_{j}$ is given by

$$
\begin{gathered}
c_{j}=\frac{1}{2 \tau}\left(1+\cos \left(\frac{j \tau}{n}\right)\right) \text { for } j \leq n \\
c_{j}=0 \quad \text { elsewhere, }
\end{gathered}
$$

where the relaxation time period $\tau$ is 0.1 days and the width of the relaxation zone $n$ is 10 grid points (about $100 \mathrm{~km}$ ). The elevation is imposed as an implicit gravity-wave radiation condition [Chapman, 1985] and the barotropic velocity uses the Flather boundary condition [Flather, 1976].

[21] The boundary conditions have been tested with ROMS for a simulation with climatological boundary values [Marchesiello et al., 2001]. Here, these boundary conditions are used to nest ROMS into the outer model NAT HYCOM [see also Barth et al., 2007].

\section{Hindcast Experiment}

[22] A 12-month model run was performed starting on 1 January 2004 and validated against in situ temperature and ADCP currents. Along with RMS error and bias, a mean square error skill score [Murphy, 1988] is used, defined by

$$
\operatorname{MSESS}=1-\frac{\operatorname{MSE}(f, x)}{\operatorname{MSE}(r, x)},
$$

where $f, x$ and $r$ represent the forecast (here the nested model), the observations, and the reference (here the outer model), respectively. If the MSESS is positive, the nested model is closer to the observations than the outer model, whereas if the MSESS is negative, the nested model is 


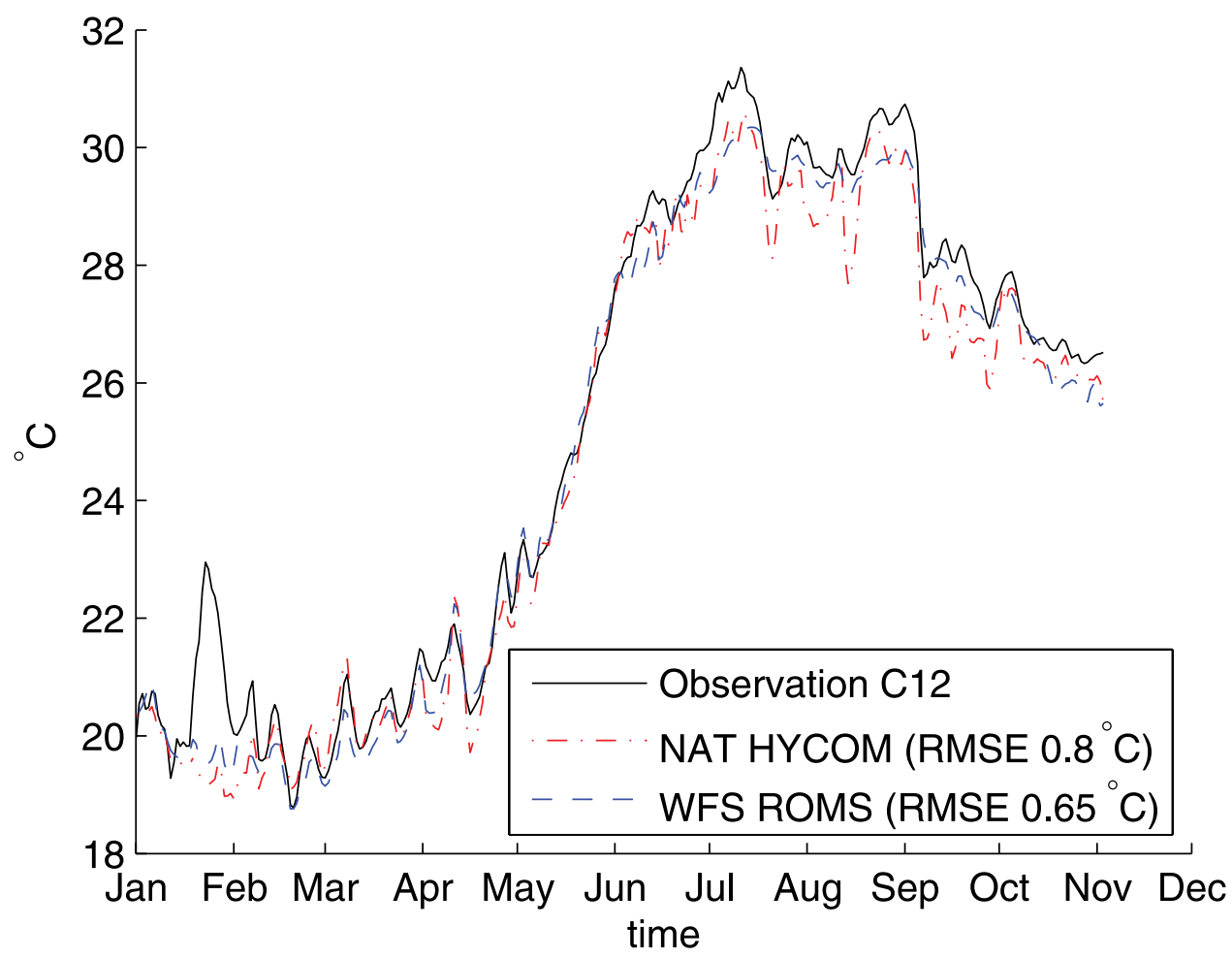

Figure 2. Temperature time series of station $\mathrm{C} 12$ at $1 \mathrm{~m}$ depth.

worse than the outer model, implying that there is no benefit in nesting the models.

\subsection{Comparison With in Situ Temperature Profiles}

[23] Figure 2 shows the time evolution of the observed surface temperature at the WFS mooring C12 (Figure 1), and the corresponding temperature of the outer and nested models. The overall agreement of both models with the observations is good. During winter (especially between mid-February and mid-April), both models capture the temperature variations at a frequency of about 10 days. These temperature changes correspond to stratification and destratification cycles induced by the wind. Owing to the atmospheric heat fluxes, the seasonal cycle and in particular the onset of the spring heating are also well represented in both models.

[24] A remarkable observation at $\mathrm{C} 12$ is the sharp temperature rise of about $3^{\circ} \mathrm{C}$ on 20 January 2004. Observed $\mathrm{SST}$ reveals that this anomalous water originates from the LC and reaches this 50-m-isobath station in the form of a filament (Figure 3). Neither ROMS nor HYCOM capture this event. From the model SST, we know that the WFS ROMS model is able of producing such filaments but not necessarily in phase with the observations. The statistical properties of filament generation are studied in section 6 .

[25] Figure 4 shows the time evolution of the observed $10 \mathrm{~m}$ depth temperature at station C14 (Figure 1) located on the $20-\mathrm{m}$ isobath along with the corresponding temperature of the nested and outer models. The main contributors to the temperature at shelf stations like $\mathrm{C} 14$ are generally the surface heat flux and the vertical mixing [He and Weisberg, 2002, 2003a]. Stratification and destratification cycles of about 10 days during winter impact the temperature down to $10 \mathrm{~m}$ depth. These variations are well reproduced by both models showing that the Mellor-Yamada 2.5 turbulence scheme of the nested model and the K-Profile Parametrization of Large et al. [1994] in the outer model produce realistic temperature distributions for these events. The role of the mixing scheme is further studied in section 4.3.

[26] During summer, the HYCOM temperature is approximately $2^{\circ} \mathrm{C}$ lower than the observed temperature. The problem also appears at other stations for observations at $5 \mathrm{~m}$ or deeper whereas this bias is not present at the surface. This indicates that the NAT HYCOM insufficiently mixes the surface temperature down to the deeper layers. Improved vertical mixing schemes and a better choice of the vertical discretization have been proposed [Halliwell, 2004] to address this problem in HYCOM in coastal regions.

[27] Table 1 shows the bias and RMS errors of the various stations at all available depths. The main conclusions of Figures 2 and 4 are also valid for other stations. The surface ( $1 \mathrm{~m}$ depth) bias and RMS error are relatively low in both models. However, for deeper measurements the RMS error of the outer model increases with depth. For most stations, a significant part of the error is due to the bias. In terms of RMS error, the nested model is better than the outer model except for station $\mathrm{C} 16$ at $1 \mathrm{~m}$. Consequently, the model skill is positive for all these locations. At C16 the skill is negative and the model nesting degrades the results at this location. The RMS error of the nested model at this station near the shelf break is essentially a model bias. The overall result gives us confidence in the nesting procedure since it confirms our expectation that a nested shelf model should be more accurate in the coastal zone than a lower resolution large-scale model.

[28] Since both models are quite close to the observed temperature at $1 \mathrm{~m}$ depth (see Table 1), the main reason for the temperature error at depth is thus the distribution of heat. 


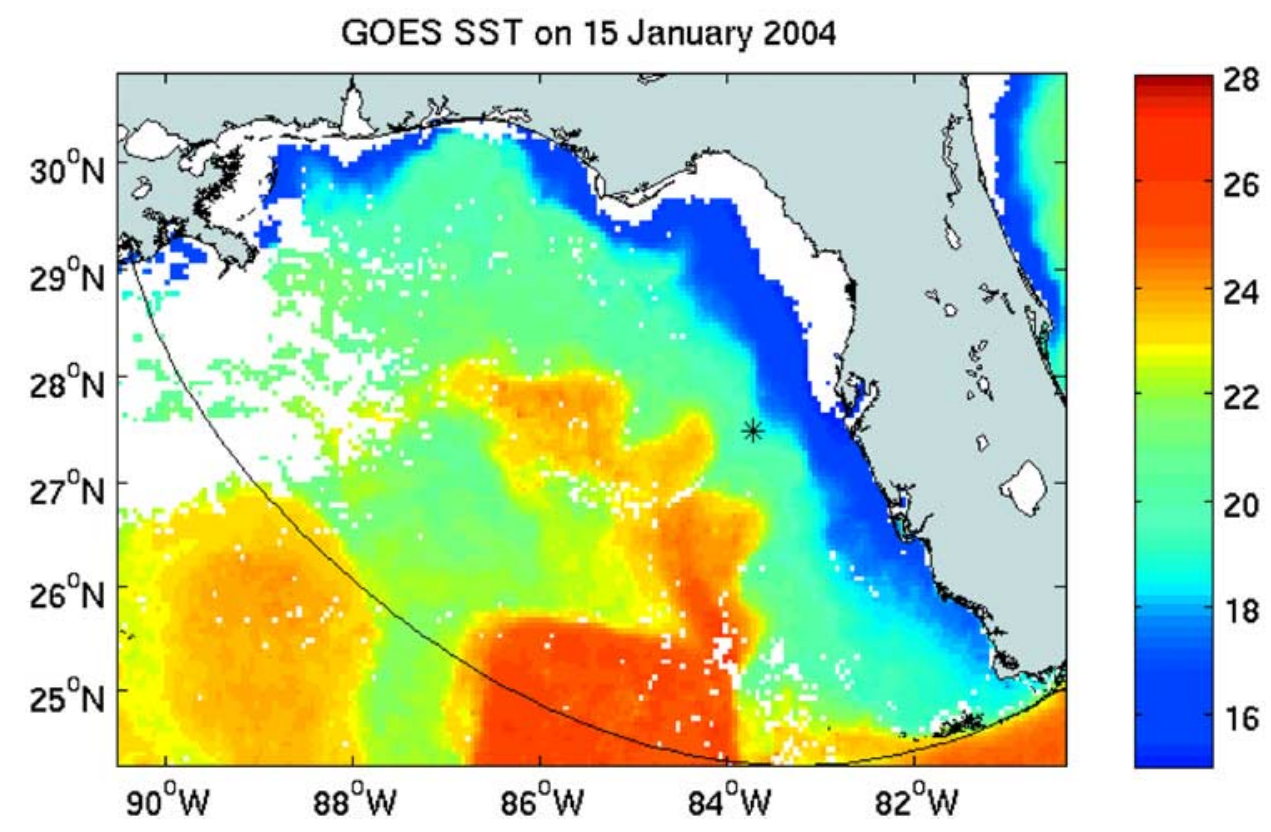

Figure 3. Filament visible in the GOES SST on 15 January 2004. The solid line marks the model boundary, and the asterisk is the position of station C12.

The nested model has a low bias during winter and summer and closely follows the seasonal temperature cycle. Shorter temperature variations generated by the atmospheric forcings are also in phase with the observed variations and are of similar amplitude.

\subsection{Comparison With in Situ Velocity Measurements}

[29] Figure 5 shows the surface velocity RMS error of different ADCP measurements for the year 2004. Tides are removed from observations with a 36-hour cutoff low-pass filter. Both models present a similar error distribution in space: RMS error for near-shore stations is lower than for offshore sites. Near-shore currents are mainly driven by winds and are thus more easily modeled than offshore currents, where deep ocean forcings play an important role. Station C16 is, as previously, quite problematic: it is located near the shelf break and the variability of this station is higher than the near-shore stations. Stations C18 and C19

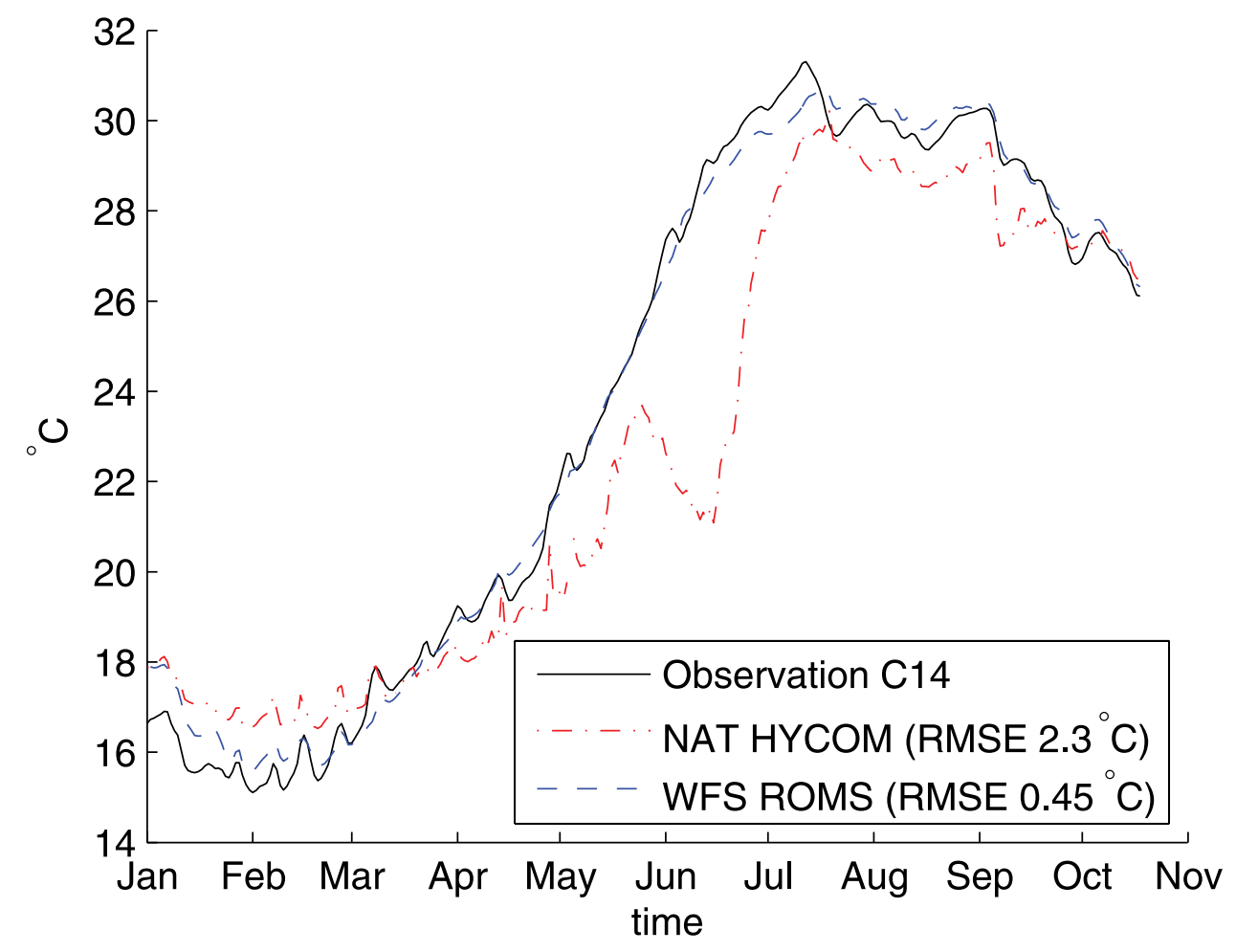

Figure 4. Temperature time series of station $\mathrm{C} 14$ at $10 \mathrm{~m}$ depth. 
Table 1. Bias and RMS Error of the Outer Model and the Nested Model Compared to in Situ Temperature ${ }^{\mathrm{a}}$

\begin{tabular}{lrrrrrr}
\hline & & \multicolumn{4}{c}{ Outer Model Nested Model } & \\
\cline { 3 - 5 } & Depth, m & Bias & RMS & \multicolumn{1}{c}{ Bias } & RMS & MSESS \\
\hline C11 & 19 & -0.62 & 1.34 & 0.38 & 0.74 & 0.70 \\
C12 & 1 & -0.50 & 0.80 & -0.36 & 0.65 & 0.34 \\
C12 & 10 & -1.05 & 1.56 & -0.38 & 0.65 & 0.83 \\
C13 & 10 & -1.24 & 1.45 & -0.67 & 0.75 & 0.73 \\
C14 & 1 & 0.18 & 0.72 & 0.04 & 0.44 & 0.63 \\
C14 & 5 & 0.07 & 0.78 & 0.02 & 0.44 & 0.68 \\
C14 & 10 & -1.02 & 2.28 & 0.09 & 0.45 & 0.96 \\
C14 & 15 & -1.82 & 3.27 & 0.34 & 0.6 & 0.97 \\
C16 & 1 & -0.67 & 1.15 & -0.99 & 1.21 & -0.11 \\
\hline
\end{tabular}

${ }^{a}$ The mean square error skill score (MSESS) of the nested model using the outer model as a reference is also shown. The record length of the stations varies but there are at least 144 days over which these statistics have been calculated. Units for RMS error and bias are ${ }^{\circ} \mathrm{C}$.

are also located near steep topography and present the highest errors. The nested model errors are almost identical to the outer model errors at those two stations, because they are located in the flow relaxation zone of the WFS ROMS. Consequently, the skill of the nested model relative to the outer model is close to zero at those stations. At station C17, the skill is negative which is related to the combination of the open boundary and the shelf break. This induces sometimes a spurious upwelling which degrades the results of the nested model.

[30] The WFS ROMS model, however, does provide improved results over the shelf. This finding is attributed to the fact that the model resolution is higher, therefore the bathymetry and coastline are more realistic. In addition, the wind forcing is more accurate near the coast since it includes in situ data.

\section{Sensitivity Experiments and Model Calibration} 4.1. Sensitivity to Heat Flux Correction

[31] We conducted a sensitivity test of the WFS model results with regards to the heat flux correction. The refer- ence simulation uses a heat flux correction strength of $\alpha=$ $-47 \mathrm{Wm}^{-2} \mathrm{~K}^{-1}$. In this simulation, a mixed layer of, for example, $\Delta z=20 \mathrm{~m}$ will converge toward the observed SST with a timescale of $\tau$, given by

$$
\tau=\frac{c_{p} \rho \Delta z}{|\alpha|}=20 \text { days }
$$

where $c_{p}$ is the heat capacity of seawater and $\rho$ its density. This can already be considered as a strong relaxation. Two additional simulations were carried out: one with a weak heat flux correction $\left(\alpha=-20 \mathrm{Wm}^{-2} \mathrm{~K}^{-1}\right)$ and one with no heat flux correction at all. The aim of these tests is to assess the model skill attributed to the relaxation to the optimal interpolated SST.

[32] Figure 6 shows the difference between the observed temperature at station $\mathrm{C} 14$ near the bottom and the model simulations with three different heat flux corrections. Without relaxation, the WFS model is generally too warm at this station. Consequently, the heat flux corrections at the surface of the two model simulations with SST relaxation generally represent a cooling of the ocean. The correction amounts to $20 \%$ and $13 \%$ of the total heat flux applied to the ocean surface for the reference simulation and the model run with weak relaxation, respectively. Table 2 gives the same error measures as Table 1 for this sensitivity test. The persistent temperature bias at all depths of station C14 reveals that the entire water column underestimates the temperature (i.e., it is not a problem of vertical temperature distribution). Since the horizontal advection of temperature on the shelf is small [Weisberg et al., 2001], one can conclude that a certain fraction of the heat flux correction is indeed an error in the atmospheric model that requires correcting through a SST relaxation.

[33] Figure 6 also reveals that the impact of the SST relaxation on the near-bottom temperature depends on the season. In fact, the sensitivity of the near-bottom temperature to the heat flux correction is the highest during winter
WFS ROMS vel. RMSE

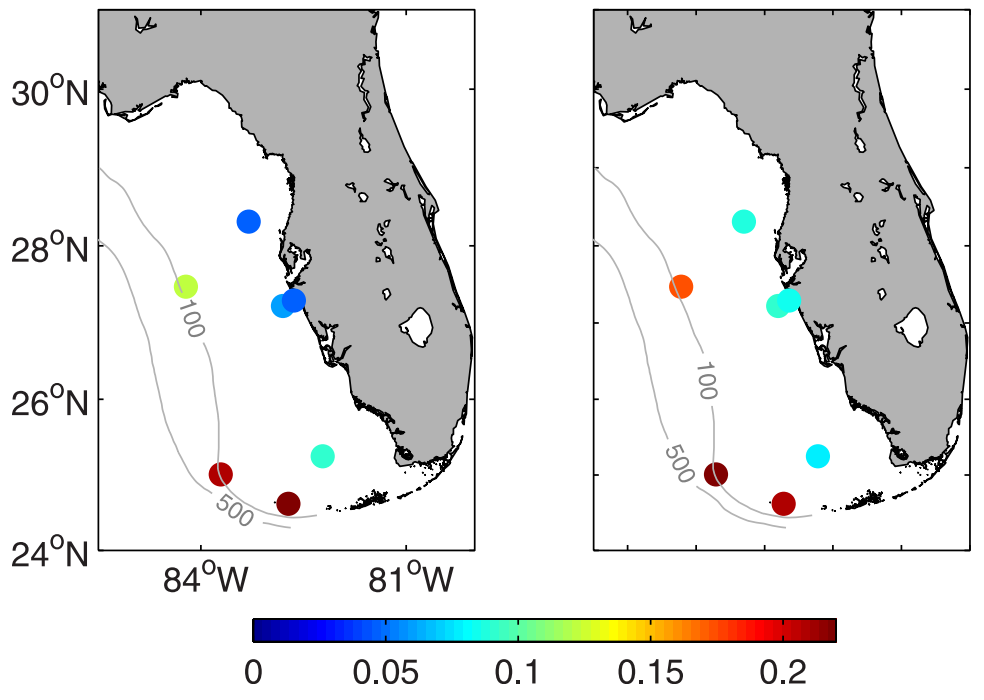

WFS ROMS vel. MSESS

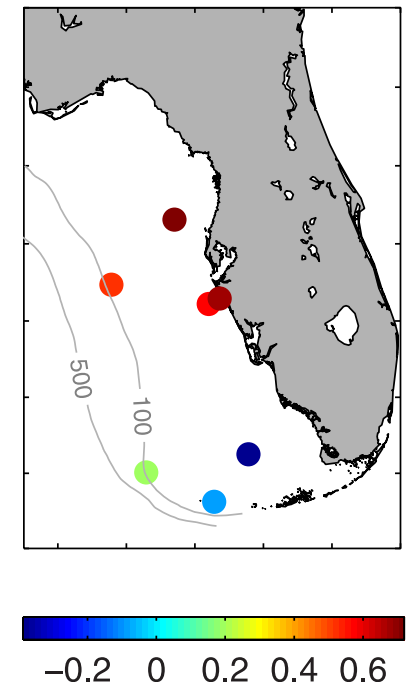

Figure 5. (left and middle) RMS error of the nested and the outer model compared to ADCP velocity measurements at the surface in $\mathrm{m} \mathrm{s}^{-1}$ and (right) the mean square error skill score. 


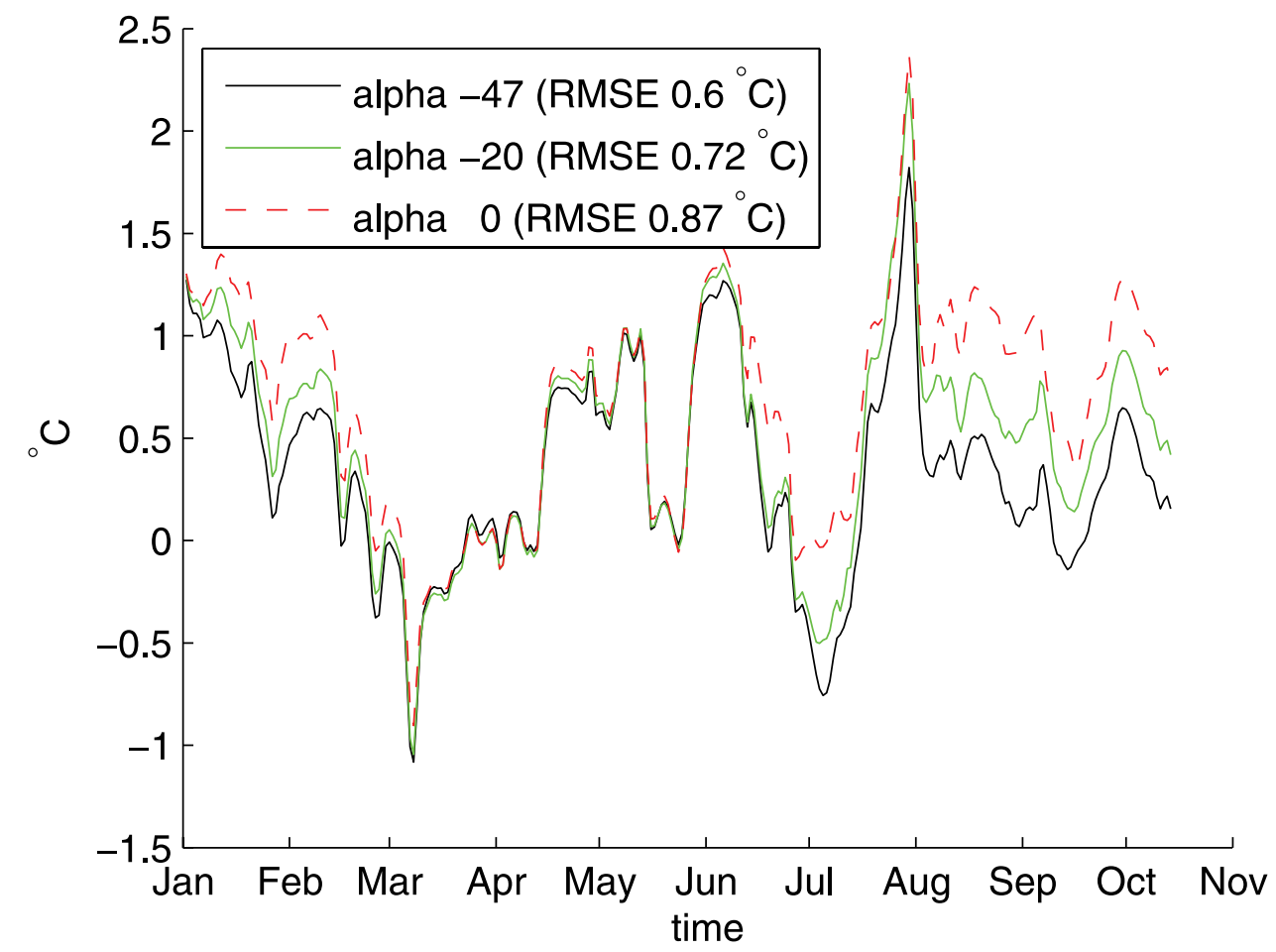

Figure 6. Difference between the observed temperature at station $\mathrm{C} 14$ at $15 \mathrm{~m}$ depth and the WFS ROMS simulation with different heat flux correction parameters $\alpha$. The units of $\alpha$ are $\mathrm{Wm}^{-2} \mathrm{~K}^{-1}$.

and autumn. During these periods the water column is weakly stratified and a SST relaxation applied at the surface affects the bottom layer through mixing. In summer, high stratification inhibits such corrective heat transfers.

[34] The SST relaxation reduces mainly the SST bias (Table 2). This is obviously related to the relaxation timescale (which depends on the mixed layer depth, since the relaxation is implemented as a heat flux correction term). Only model errors with a timescale longer than the relaxation timescale are affected by the heat flux correction, as it can be shown easily by considering the effect of the relaxation term in the temperature equation,

$$
\frac{d T}{d t}=\frac{1}{\tau}\left(T^{o}-T\right)
$$

where $T$ and $T^{o}$ are the model and observed SST respectively and $\tau$ is the relaxation timescale. If we take the Fourier transform $\mathcal{F} T=\int_{-\infty}^{\infty} T(t) e^{-i \omega t} d t$ of both sides of equation (9), we obtain, after some rearrangement, the frequency response of the relaxation term,

$$
\mathcal{F} T=\frac{1}{1+i \omega \tau} \mathcal{F} T^{o}
$$

Frequencies higher than $1 / \tau$ are only weakly affected by the SST relaxation, while at lower frequencies the variations are close to the observations. The fact that the coefficient in the last equation is complex reminds us that the relaxation also introduces a retardation effect, i.e., a phase shift between the model and the observations. SST relaxation acts therefore as a low-pass filter with a cutoff frequency directly related to the relaxation strength. Consequently, the RMS error reduction due to relaxation is mainly a consequence of the reduction of the bias (Tables 1 and 2).

\subsection{Sensitivity to Wind Forcing}

[35] The model results presented in section 3.1 use a wind field that merges NCEP winds with in situ wind observations using OI. In an attempt to assess the influence of the wind forcing on the velocities of WFS ROMS, we repeated the regional simulation with NOGAPS wind forcing which is also used by the large-scale model. Without model nesting, the beneficial impact of the OI winds on the shelf circulation has been already demonstrated [He et al., 2004]. Here we want to reassess the benefit of the OI winds in the context of a nested model. One might expect the same outcome for this case. On the other hand, the use of different wind forcings might lead effectively to a discontinuous

Table 2. Bias and RMS Error of WFS ROMS With Weak

\begin{tabular}{|c|c|c|c|c|c|}
\hline & & & & $\alpha=0$ & ${ }^{-2} \mathrm{~K}^{-1}$ \\
\hline & Depth, m & Bias & RMS & Bias & RMS \\
\hline C11 & 19 & -0.57 & 0.84 & -0.84 & 1.03 \\
\hline $\mathrm{C} 12$ & 1 & 0.39 & 0.69 & 0.41 & 0.75 \\
\hline $\mathrm{C} 12$ & 10 & 0.44 & 0.70 & 0.50 & 0.78 \\
\hline C13 & 10 & 0.65 & 0.72 & 0.76 & 0.84 \\
\hline C14 & 1 & -0.22 & 0.51 & -0.49 & 0.68 \\
\hline $\mathrm{C} 14$ & 5 & -0.20 & 0.52 & -0.46 & 0.67 \\
\hline C14 & 10 & -0.26 & 0.55 & -0.50 & 0.70 \\
\hline $\mathrm{C} 14$ & 15 & -0.49 & 0.72 & -0.68 & 0.86 \\
\hline C16 & 1 & 1.06 & 1.30 & 1.25 & 1.53 \\
\hline
\end{tabular}
Relaxation and No Relaxation Compared to in Situ Temperature ${ }^{a}$

${ }^{\mathrm{a}}$ The record length of the stations varies, but there are at least 144 days over which these statistics have been calculated. Results of the reference run $\left(\alpha=-47 \mathrm{Wm}^{-2} \mathrm{~K}^{-1}\right)$ are given in Table 1 . 
Filtered ADCP currents $(\mathrm{m} / \mathrm{s}) \mathrm{C} 14$ at $-4 \mathrm{~m}$

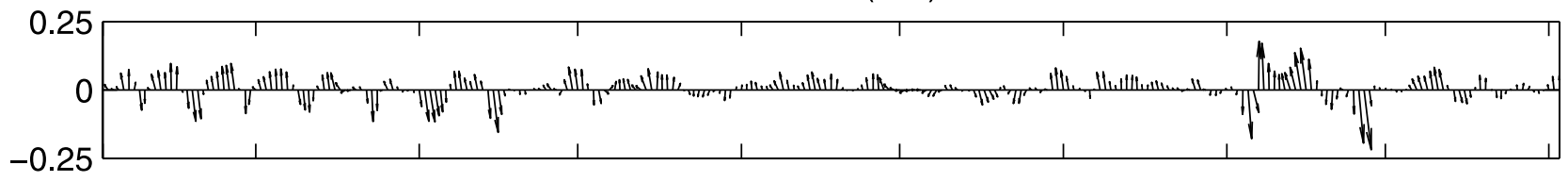

WFS ROMS with OI-winds, $\gamma=0.686, \phi=-14, r=0.592$, RMS $=0.046 \mathrm{~m} / \mathrm{s}$

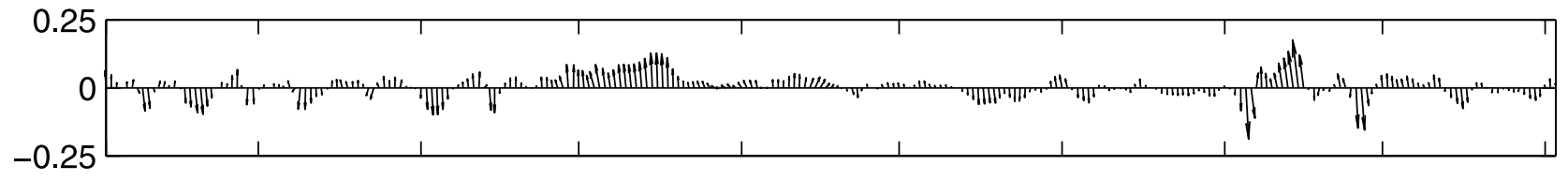

WFS ROMS with NOGAPS winds, $\gamma=0.703, \phi=-17, r=0.763, \mathrm{RMS}=0.051 \mathrm{~m} / \mathrm{s}$

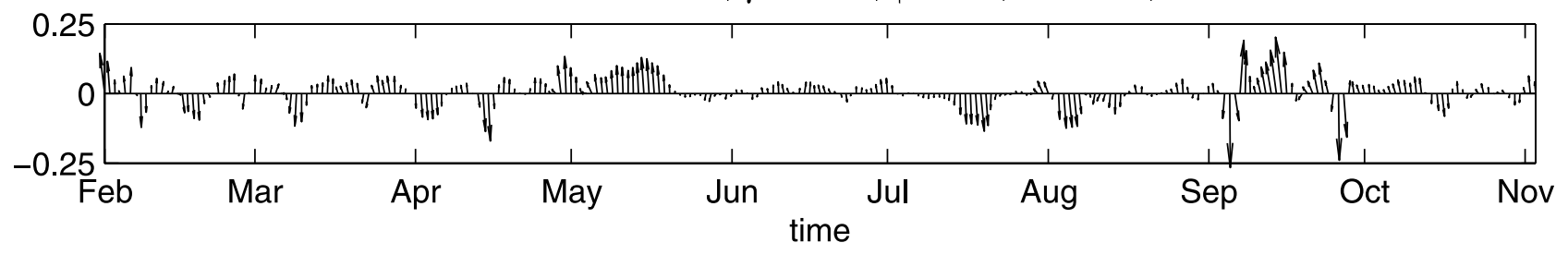

Figure 7. Near-surface (4 $\mathrm{m}$ depth), 36-hour filtered currents at station C14. The three panels show the observations, WFS ROMS with OI winds and WFS ROMS with NOGAPS winds. The error measures are computed using $u$ and $v$ components as real and imaginary parts of a complex time series [Kundu and Allen, 1976]. Here $\gamma$ and $\phi$ are the amplitude and phase (in degrees) of the complex correlation coefficient. The parameter $r$ is the amplitude of the complex regression coefficient. The RMS error is computed using also both velocity components.

wind forcing at the model open boundary that arguably one should seek to avoid. The discontinuity of the wind speed by using the OI wind has been computed as the RMS difference (averaged over time and over the open boundary) between the HYCOM wind forcing and the OI winds. In average, the difference is $3 \mathrm{~m} / \mathrm{s}$ which amounts to $28 \%$ of the variance of the NOGAPS wind speed. The discontinuity of the wind field is thus a nonnegligible fraction of the variability of the wind field.

[36] Figure 7 shows the observed near-surface currents at the 20-m-isobath station C14, detided with a 36-hour lowpass filter, the corresponding model currents of the WFS ROMS forced by OI winds and the WFS ROMS forced by NOGAPS winds. At this station, the error reduction due to the use of the OI winds accounts for $0.5 \mathrm{~cm} \mathrm{~s}^{-1}$. At most other surface stations (4 out of 6), we generally noticed an improvement of about $10 \%$ using the OI winds in terms of RMS error reduction.

[37] The regression coefficient ( $r$, included in Figure 7) shows that the model generally underestimates the current strength. The OI winds degrade in fact the regression coefficient but they improve the direction of the flow since the phase of the complex correlation coefficient is reduced. This indicates that the OI winds are of weaker magnitude but with an improved direction and in this case the improvement in current direction outweighs the degradation in speed, since the total RMS error is reduced.

\subsection{Impact of the Mixing Scheme and Vertical Resolution}

[38] The 2004 model run was repeated with the K-profile parametrization [KPP Large et al., 1994] in order to determine whether the KPP or the Mellor Yamada level 2.5 (MY) turbulence scheme is more appropriate to model the WFS.

[39] Station C14 is well suited to compare the mixing schemes, since four sensors (at 1, 5, 10 and $15 \mathrm{~m}$ depth) were operational in 2004 at this location. At other stations the vertical resolution of the observations is lower. In addition, horizontal currents at this station are relatively small, thus the vertical mixing of heat, salt and momentum is the dominant source of variability.

[40] In order to highlight the synoptic temperature variability at this station, the seasonal cycle shown in Figure $8 \mathrm{~b}$ has been removed from all temperature time series in Figures $8 \mathrm{c}-8 \mathrm{f}$ at all depths. The seasonal cycle is obtained by fitting a cosine function to the depth-averaged observed temperature.

[41] The observations are shown in Figure 8c. From January to May, the temperature stratification is quite small. The stratification only builds up when the winds are weak ( 2 days low-pass filtered winds are shown in Figure $8 \mathrm{a}$ ), but it is eroded as soon as the wind increases. After the onset of the spring heating (in May), a summer stratification is observed. In this period, the influence of the wind on the stratification is much smaller. In July, the stratification collapses for the rest of the time series except for a remarkable event around 30 July 2004 where the bottom temperature suddenly decreases by $1.3^{\circ} \mathrm{C}$.

[42] The corresponding model time series of the MY closure scheme and the KPP scheme are shown in Figures 8d and 8e, respectively. Both schemes simulate reasonably well the wind-induced destratification events (from January to April) and the corresponding heat loss. However, the observations 
(a) Wind

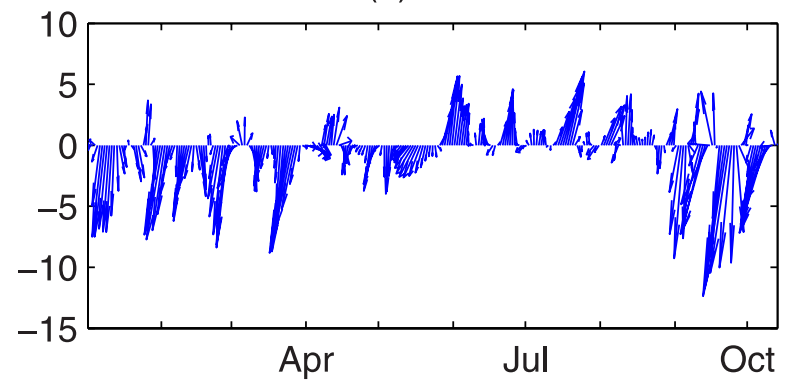

(c) Observations

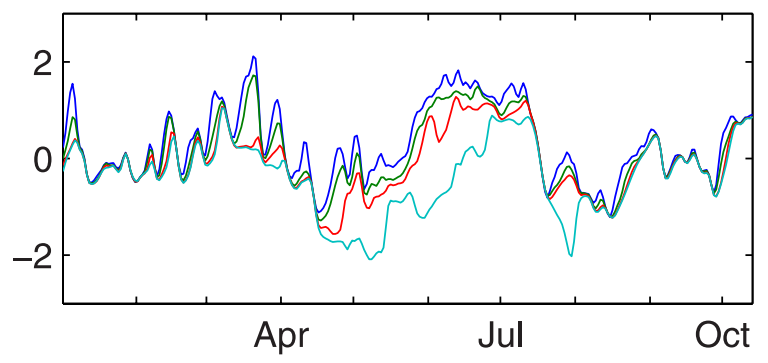

(e) ROMS KPP-32 levels

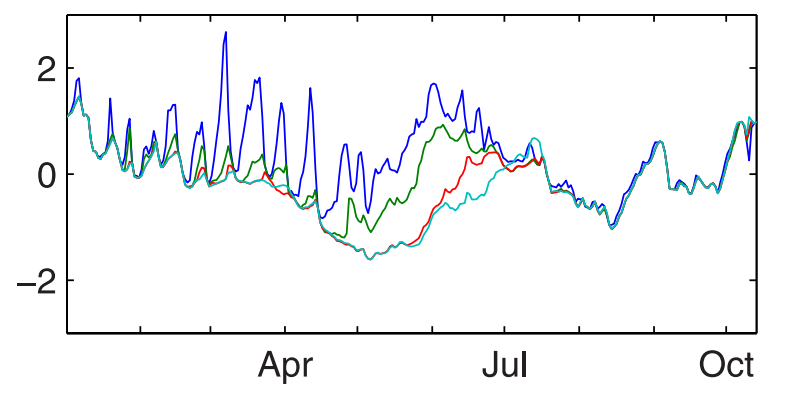

(b) Seasonal cycle

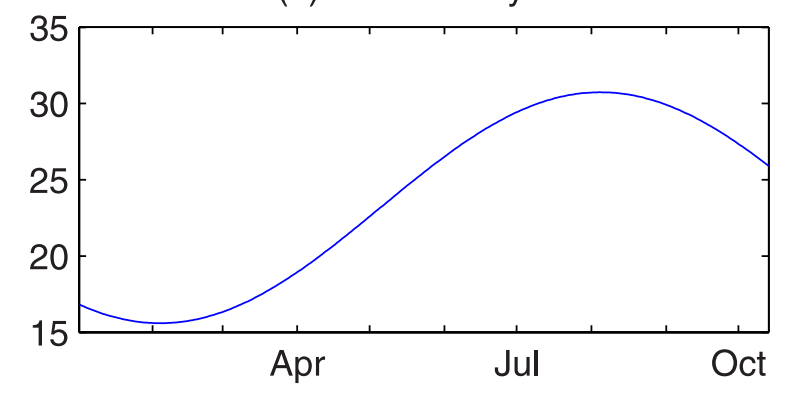

(d) ROMS MY-32 levels

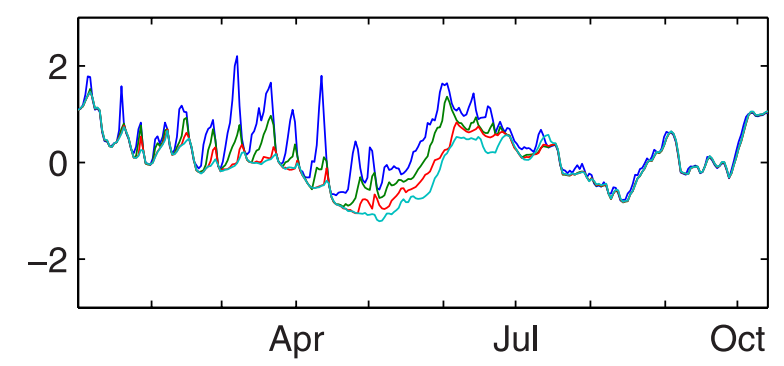

(f) ROMS MY-16 levels

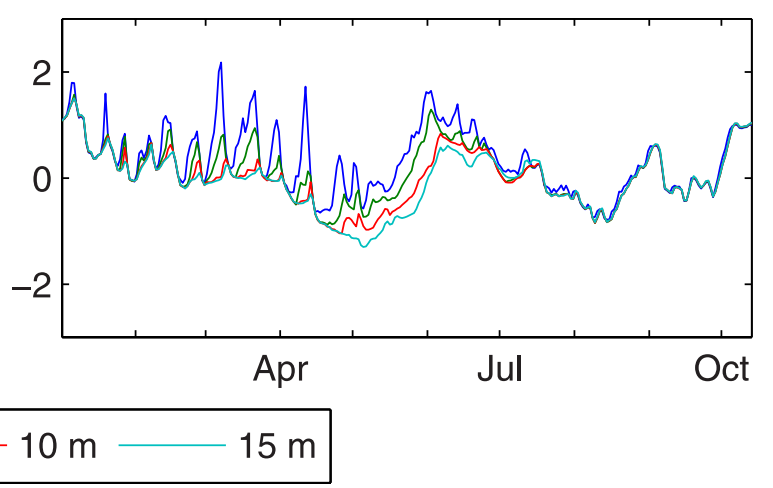

Figure 8. Sensitivity of the model temperature to the mixing scheme (KPP or MY) and the vertical resolution. Temperature is expressed in ${ }^{\circ} \mathrm{C}$ and winds in $\mathrm{m} \mathrm{s}^{-1}$. The seasonal cycle shown in Figure $8 \mathrm{~b}$ is obtained by fitting the depth-averaged temperature to a cosine. This seasonal cycle is subtracted from all temperature time series in Figures $8 \mathrm{c}-8 \mathrm{f}$.

show that the temperature variations at $5 \mathrm{~m}$ are of similar magnitude than the variations at the surface. In the model simulations with the KPP mixing scheme, the temperature increase at $5 \mathrm{~m}$ during calm periods is largely underestimated. The temperature increase for the MY scheme at this depth is more realistic but still smaller than the observations.

[43] Durski et al. [2004] compared both mixing schemes extensively for application in the coastal ocean under different wind forcings and stratification. In our simulations, KPP entrains less in winter than MY. This agrees with the findings of Durski et al. [2004]. The authors found that KPP mixes less than MY under weak stratification and for a pulsed wind forcing.

[44] After May, KPP represents the overall temperature difference between the surface and the bottom layer better than the MY scheme. In the later, the temperature difference is too low. This is also consistent with the results of Durski et al. [2004]. At high stratification and under a steady wind stress, MY mixes more than KPP. Therefore, the thermocline from May to July is stronger with KPP than with MY and the variations of the wind stress from May to July are indeed smaller than the wind stress variations from January to April.

[45] Unfortunately, no model simulation reproduces the abrupt temperature decrease around 30 July 2004. One may speculate that this phenomenon corresponds to a horizontal advection of cold bottom water, since it is unrelated to the temperature of the surface layer. After this event, KPP and MY produce a well mixed water column in agreement with the observations.

[46] On the basis of these comparisons, MY seems to work better during winter, when the momentum flux is important and the heat flux is negative (directed from the ocean to the atmosphere) and KPP produces better results 
from May to July when the heat flux goes into the ocean and winds are weaker. After August, when the water column is completely homogeneous, both mixing schemes perform equally well.

[47] We computed the total RMS error (averaged over time and depth) for each experiment. The total RMS error of the MY mixing scheme is with $0.49^{\circ} \mathrm{C}$, slightly lower than the total RMS error of KPP with $0.52^{\circ} \mathrm{C}$. In average, the results obtained by the MY scheme are thus closer to the observed temperature at $\mathrm{C} 14$ than the results by KPP. The statistical significance of this error is examined with a student t-test. The square difference between the observations $T_{n}^{o}$ and the model temperature with MY mixing scheme $T_{n}^{M Y}$ and with KPP mixing scheme $T_{n}^{K P P}$ are defined as

$$
\begin{gathered}
d_{n}^{M Y}=\left(T_{n}^{M Y}-T_{n}^{o}\right)^{2} \\
d_{n}^{K P P}=\left(T_{n}^{K P P}-T_{n}^{o}\right)^{2},
\end{gathered}
$$

where $n$ is the time index. The mean of those time series is obviously the square of the RMS error. The time series $x_{n}$ is introduced to determine if these means are significantly different,

$$
x_{n}=d_{n}^{M Y}-d_{n}^{K P P} .
$$

Assuming that $x_{n}$ follows a Gaussian distribution, which is approximately the case, the quantity $t$ follows a student $t$ distribution if the RMS error of both model simulations are the same (null hypothesis),

$$
t=\frac{\sqrt{N}|\bar{x}|}{s}
$$

where $\bar{x}$ and $s$ are the mean and the standard deviation of the time series $x_{n}$ and $N$ is its length. In our case, $t$ equals 3.70 , which exceeds the threshold of 1.96 for a significance level of $5 \%$ which leads to the rejection of the null hypothesis. Thus the RMS error of MY scheme is significantly lower than the RMS error of the KPP scheme at station C14. This conclusion also holds when the total RMS error is extended to include the model error at all other shelf stations (namely C11, C12, C13, C15, C16 and C19).

[48] All model simulations use 32 terrain-following levels with a finer resolution at the surface and bottom than in the interior of the water column. In order to assess the importance of the resolution, the model simulation was repeated with 16 vertical levels using the MY scheme. The temperature at $\mathrm{C} 14$ (without the seasonal cycle) is shown in Figure 8f. Qualitatively, the results are very similar to those obtained with a higher vertical resolution. The different turbulent regimes are well reproduced in this experiment. The average RMS error at this station is $0.51^{\circ} \mathrm{C}$ and is thus larger than the RMS error obtained with 32 levels $\left(0.49^{\circ} \mathrm{C}\right)$. Despite the difference in RMS error is small, it is statistically significant (using the same procedure as above). This leads to the conclusion that with a lower vertical resolution, the model is able to qualitatively reproduce the different mixing regimes in agreement with the observations but the skill of the model in terms of average RMS error is improved when the resolution is increased.

\subsection{Sensitivity to Horizontal Resolution and Bathymetry}

[49] In this section, the sensitivity of the model solution to the horizontal resolution is assessed. The model grid used previously is referred hereafter as the "coarse" grid. Increased resolution can improve the model solution in two different ways: either the spatial scales of variability inherent to the ocean are better resolved or the spatial variation of the bathymetry are better represented. The spatial scales in other forcing fields such as atmospheric fields are in most cases already much larger than the typical horizontal resolution of regional ocean models and resolution is thus not a limiting factor for atmospheric fields.

[50] The coarse model grid is refined by a factor of 2; that is, every model grid cell is divided in $2 \times 2$ grid cells. In a first experiment, the coarse bathymetry is simply linearly interpolated to the high-resolution grid. The variations of the bottom topography from one grid cell to a neighboring grid cell are thus smaller than those variations in the coarse model bathymetry. The $r$ factor of this bathymetry is

$$
r=\max _{i, j}\left[\max \left(\left|\frac{h_{i, j}-h_{i+1, j}}{h_{i, j}+h_{i+1, j}}\right|,\left|\frac{h_{i, j}-h_{i, j+1}}{h_{i, j}+h_{i, j+1}}\right|\right)\right]=0.29 \text {. }
$$

In a second experiment, the model bathymetry is directly regenerated from the bathymetric database at the highresolution model grid. This bathymetry (called hereafter the "fine" bathymetry) is smoothed such that the $r$ factor of this bathymetry is 0.55 which is identical to the $r$ factor of the coarse model bathymetry. This factor plays an important role in the accuracy of the pressure gradient formulation of models with terrain-following coordinates.

[51] Both high-resolution configurations are integrated for one year and the RMS error relative to the surface ADCP current measurements are computed. Since the primary interest now is the improvement or degradation relative to the coarse model results, the mean square error skill scores are calculated using the coarse model results as the baseline (in Figure 5, the HYCOM results were used as the baseline). These skill scores are shown in Figure 9. Surprisingly, the model solution is mostly insensitive to the increase of the horizontal resolution using only the interpolated bathymetry (Figure 9, left). Except for the large degradation at station $\mathrm{C} 18$, the skill score is close to zero. This indicates that the coarse model grid already resolves well the spatial structures especially on the shelf.

[52] A clear improvement can however be seen with the model simulation using the fine bathymetry (Figure 9, right). The model velocity is improved in particular near the shelf break (the 100-m isobath).

[53] These experiments indicate that a finer model grid does improve the model solution because it admits a more representative bathymetry and not because the inherent spatial scales of the flow are better resolved (since they are already well resolved in the coarse resolution grid).

[54] The sea surface height standard deviation of the coarse grid model, the fine grid model (with fine bathymetry) and AVISO SSH altimetry [Le Traon et al., 1998; Dorandeu and Le Traon, 1999] are also computed 
vel. MSEES with interpolated bath.

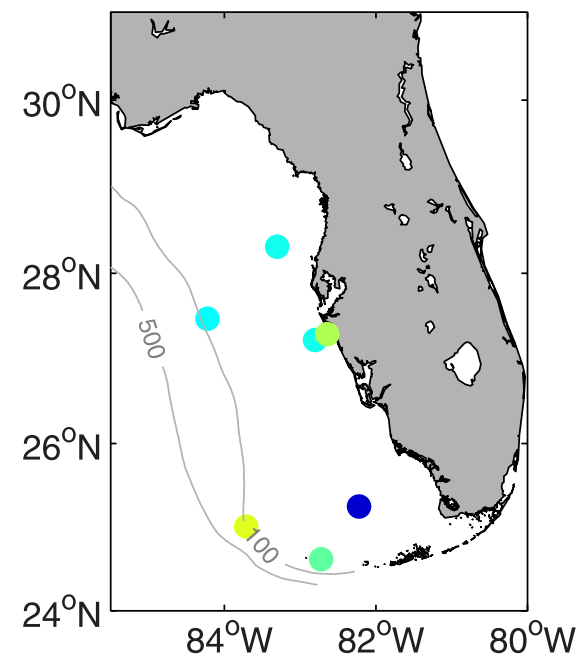

vel. MSEES with finer bath.

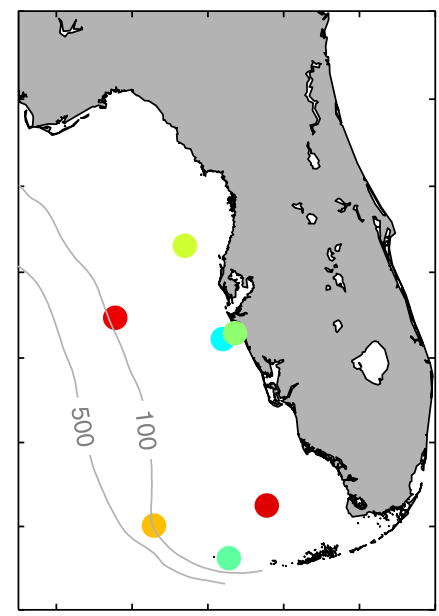

0.2

0.4

Figure 9. The surface-velocity mean square error skill score (left) of the model with two-times increased horizontal resolution and interpolated bathymetry and (right) of the model with increased horizontal resolution and finer bathymetry.

(Figure 10). The model solution is averaged over every 3.5 days to match the time sampling of the AVISO SSH observations. Interestingly, the magnitude and distribution of the coarse grid and fine grid model SSH standard deviation are very similar. This indicates that the SSH variability is mainly introduced into the model through the open boundary and that the mesoscale variability in the coarse grid model is already well resolved. In the present case, the statistics of the model variability do not depend on the model resolution. The model SSH standard deviation agrees also with the standard deviation based on altimetry. In particular, the presence and location of the two variability maxima and the spatial extent of the SSH variance is well represented by the model simulations. However, the standard deviation of the observations is almost everywhere slightly higher than the model standard deviation. This might come in part from the fact that error (due to incompletely removed tides, for example) increase the standard deviation of the SSH fields derived from altimetry. This comparison shows that the result of the coarse resolution model in a statistical sense are already adequate and that not much is gained by increasing the model resolution.

[55] In summary, these calibration experiments show that the choice of the wind forcing (a special WFS wind product including observations or the NOGAPS wind), the SST relaxation strength, the mixing scheme (MY or KPP) and vertical resolution adopted in the model configuration of section 2 are appropriate and that the statistics of the model
Coarse grid model
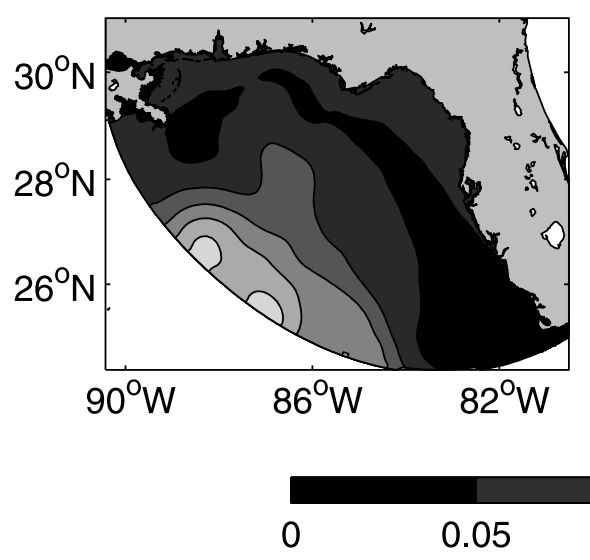

Fine grid model

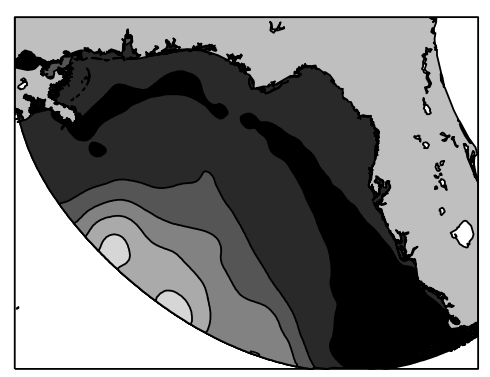

Observations

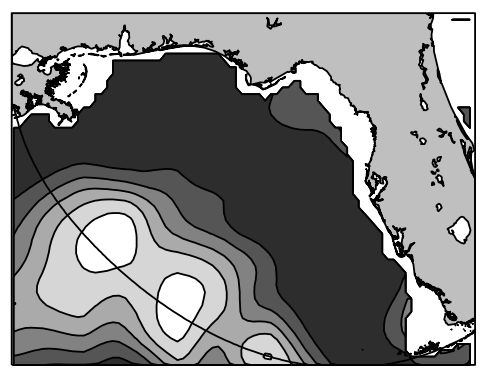

\section{1}

0.15

0.2

0.25 0.3

Figure 10. The sea-surface height standard deviation for 2004 of the coarse-grid model, the fine-grid model (with high-resolution bathymetry) and observations. 


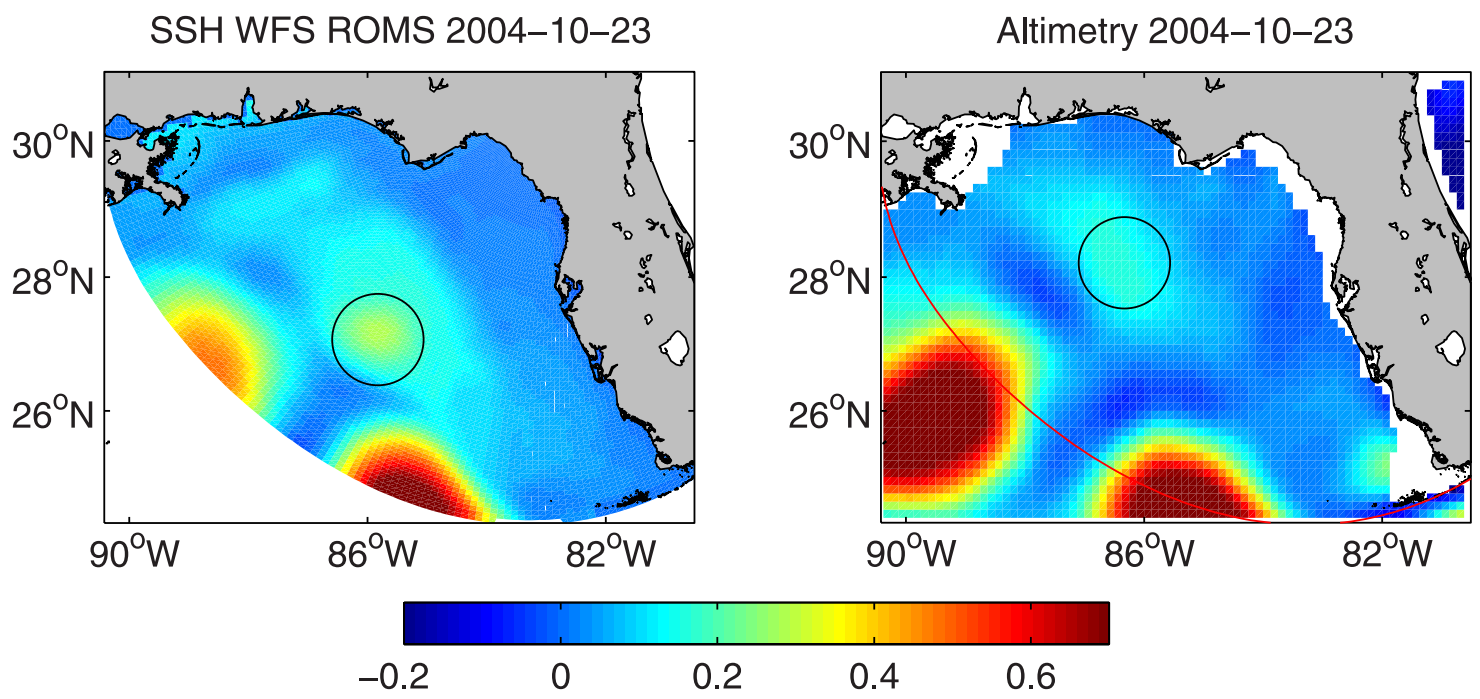

Figure 11. (left) WFS ROMS absolute sea surface height and (right) observed sea level anomaly added to the mean SSH from NAT HYCOM on 23 November 2004. The circle shows the approximate position of the vortex.

solution do not change much by increasing the model resolution.

\section{Propagation of an Anticyclonic Vortex}

[56] With this calibrated model we will now address some process-oriented questions concerning the mesoscale flow generated by the LC. In this section the movement of an anticyclonic vortex generated by the $\mathrm{LC}$ is examined.

[57] Figure 11 (left) shows an anticyclonic vortex $\left(28^{\circ} \mathrm{N}\right.$, $87^{\circ} \mathrm{W}$ ) in the WFS model solution. This vortex was created from warm water detaching in form of filaments from the LC with the help of cyclonic LC Frontal Eddies. Such processes provide a mechanism to transfer nutrient-rich, river-derived water to offshore or occasionally to the outer WFS [Hamilton and Lee, 2005]. After the anticyclone is separated from the $\mathrm{LC}$, it moves northwestward until reaching the DeSoto canyon region (Figure 1). Eddies in the DeSoto canyon play an important role in advecting cold and nutrient-rich water masses onto the shelf [Weisberg and He, 2003].

[58] The presence of this eddy is confirmed by satellite altimetry (Figure 11, right). The vortex in the WFS ROMS model is stronger than the observed eddy and lags about 12 days behind. However, the trajectory and speed of the vortex are comparable in both cases: after detachment from the LC, the vortex reaches a latitude of $28^{\circ} 30^{\prime} \mathrm{N}$ after 35 days in the model, while the observed eddy covers the same distance in 28 days.

[59] The mean advective flow has been calculated using a time and space average of the model velocity spanning the duration of the eddy propagation phase (35 days) and the region crossed by the eddy. Although the background currents flow southeastward at $1.7 \mathrm{~cm} \mathrm{~s}^{-1}$, the vortex as simulated by the WFS ROMS model moves in the opposite direction at $7.3 \mathrm{~cm} \mathrm{~s}^{-1}$. The propagation speed calculated from altimetry is $9.1 \mathrm{~cm}^{-1}$.

[60] To explain the propagation of the vortex, we examined the ambient potential vorticity. The isopycnal surface corresponding to the density of $1025 \mathrm{k} \mathrm{gm}^{-3}$ separates the upper layer containing the eddy from the deep ocean. Figure 12 shows the depth of this layer based on the annual average temperature and salinity. This depth is strongly affected by mesoscale activity in the surface layer and a shorter averaging time period appeared to be insufficient to smooth the mesoscale variability out. The ROMS results provide the density as a function of depth. By linear interpolation of the inverse of this function, the depth of this isopycnal is determined. The direction of the vortex propagation agrees indeed with the slope of this layer: fluid parcels are squeezed as they move toward the WFS, decreasing their relative vorticity; and they are stretched as they move away from the WFS, increasing their relative vorticity. The net effect of these anomalies is to drive the core of the eddy northwestward. On the basis of the reduced gravity approximation, Cushman-Roisin [1994] derived the propagation speed $\mathbf{u}$ of a vortex under these circumstances:

$$
\mathbf{u}=\mathbf{u}_{0}-\frac{g^{\prime}}{f} \nabla h \Lambda \mathbf{e}_{z},
$$

where $\mathbf{u}_{0}$ is the velocity vector of the background flow, $g^{\prime}$ is the acceleration due to the reduced gravity, $f$ is the Coriolis parameter, $h$ is the depth of the surface layer, and $\mathbf{e}_{z}$ is the unit vector pointing upward. The numerical values for the anticyclonic vortex are estimated from the WFS ROMS results,

$$
\begin{gathered}
\left\|u_{0}\right\|=1.7 \mathrm{cms}^{-1} \\
g^{\prime}=0.019 \mathrm{~ms}^{-2} \\
\|\nabla h\|=3.10^{-4} .
\end{gathered}
$$

[61] The theoretical propagation speed from equation (16) is $6.7 \mathrm{~cm} \mathrm{~s}^{-1}$. Given the idealized context in which 


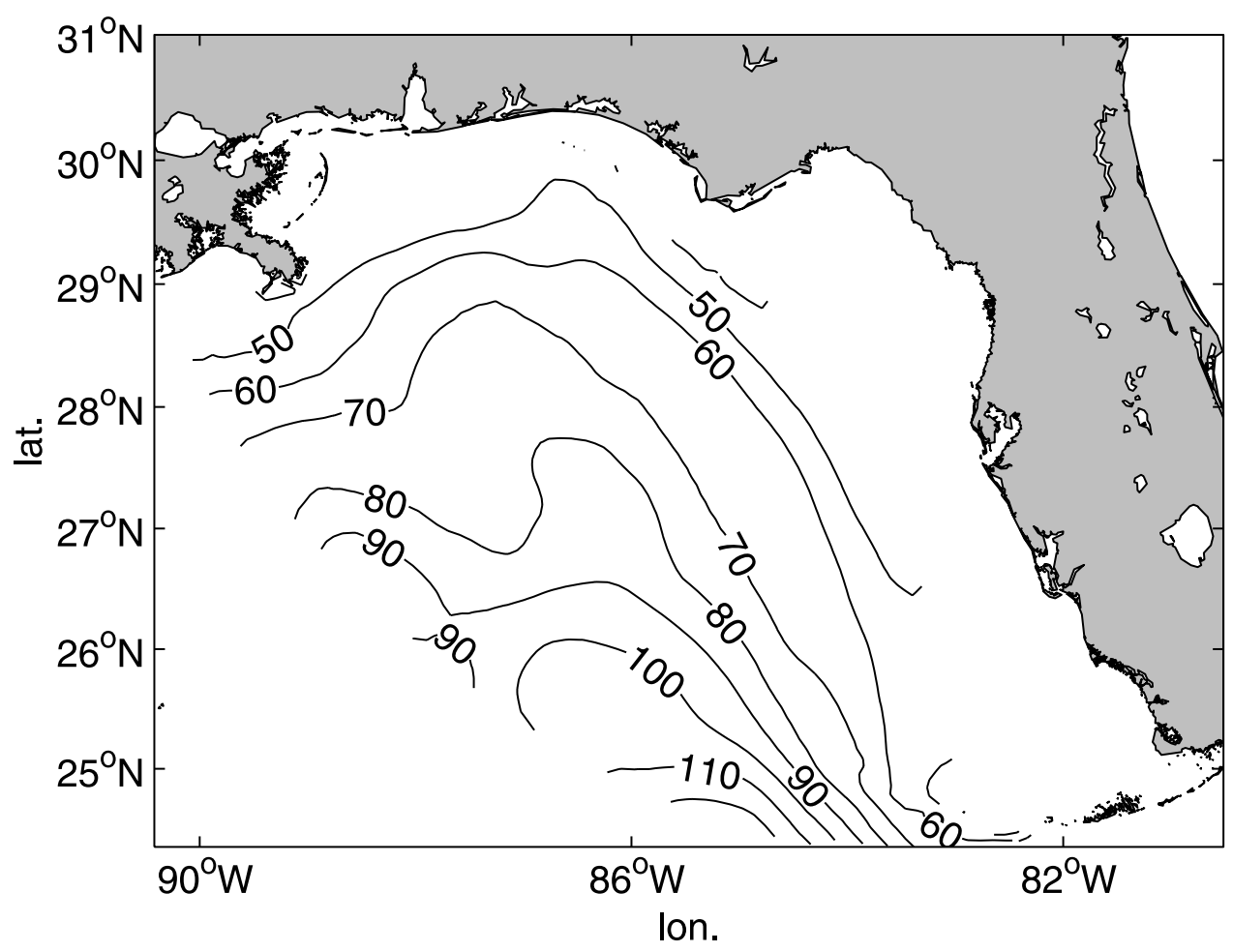

Figure 12. Depth of the $1025 \mathrm{~kg} \mathrm{~m}^{-3}$-isopycnal surface computed from the model temperature and salinity, averaged over 1 year.

equation (16) is derived, this value is in good agreement with the propagation speed of the vortex in the model $\left(7.3 \mathrm{~cm} \mathrm{~s}^{-1}\right)$ and also comparable to the vortex speed in the altimetry $\left(9.1 \mathrm{~cm} \mathrm{~s}^{-1}\right)$. This shows that the slope of the isopycnal surface coupled with the conservation of potential vorticity is responsible for the propagation of the vortex. The propagation due to advection appears to be negligible compared to the velocity component due to the gradient of ambient potential vorticity.

[62] As a corollary, the fact that the model vortex speed agrees with the speed derived from altimetry is an indirect validation of the slope of the isopycnal surface and density difference between deep ocean and surface layers. We also see that the ambient vorticity gradient is not only due to bottom topography, but also due to the background density structure which further explains why we see the generation of filaments and eddies far away from the shelf break.

\section{Tracer Experiment}

[63] In the previous section, we studied an isolated vortex of LC water which moved toward the De Soto Canyon where it disintegrated. A fraction of the LC water was eventually advected onto the shelf. In this section we examine these processes where LC water reaches the shelf from a statistical perspective. In particular, we want to determine the overall quantity of LC water that reaches the shelf and the process by which this occurs. As a practical application, the fronts derived from LC water are sometimes associated with the concentrations of the red tide organism, Gymnodinium breve, off the west Florida shelf [Tester and Steidinger, 1997]. The LC water itself is depleted of nutrients, but their density anomaly and the associated currents help to advect nutrient-rich deep water through the bottom Ekman layer [Weisberg and He, 2003]. Flow features generated by the LC play thus an important, albeit indirect, role in advecting nutrients from the deep ocean onto the shelf.

[64] LC water differs from the surrounding water masses owing to its high temperature and, to a lesser degree, to its higher salinity. However, surface temperature is only a good indication of LC water during winter and autumn. In summer, surrounding water masses heat up and the temperature can no longer be reliably used to distinguish the origin of the surface water. The discharge of the Mississippi River makes the identification of the LC water based on salinity also a difficult task. In order to track the LC water in the WFS ROMS model we introduce a passive tracer which is 1 for LC water and 0 otherwise. We provided a boundary and initial condition for this tracer based on the NAT HYCOM field. The identification of the LC water is based on the surface elevation and the salinity. If the NAT HYCOM's surface elevation exceeds $17 \mathrm{~cm}$ and if its salinity is higher than 35.3 then the corresponding fluid parcel in the boundary and initial conditions is identified as LC water. The criterion based on surface elevation identified the horizontal extent of the LC. This threshold was also used by Leben [2005] to define the LC. The salinity criterion limits essentially the vertical extent of the LC. The nesting procedure of this tracer is identical to the nesting of temperature and salinity explained in section 2.4 .

[65] We performed a 2-year simulation with this tracer starting 1 January 2004, as previously using the model configuration based on the sensitivity analysis. Since the tracer is passive, the results of the physical model are not 


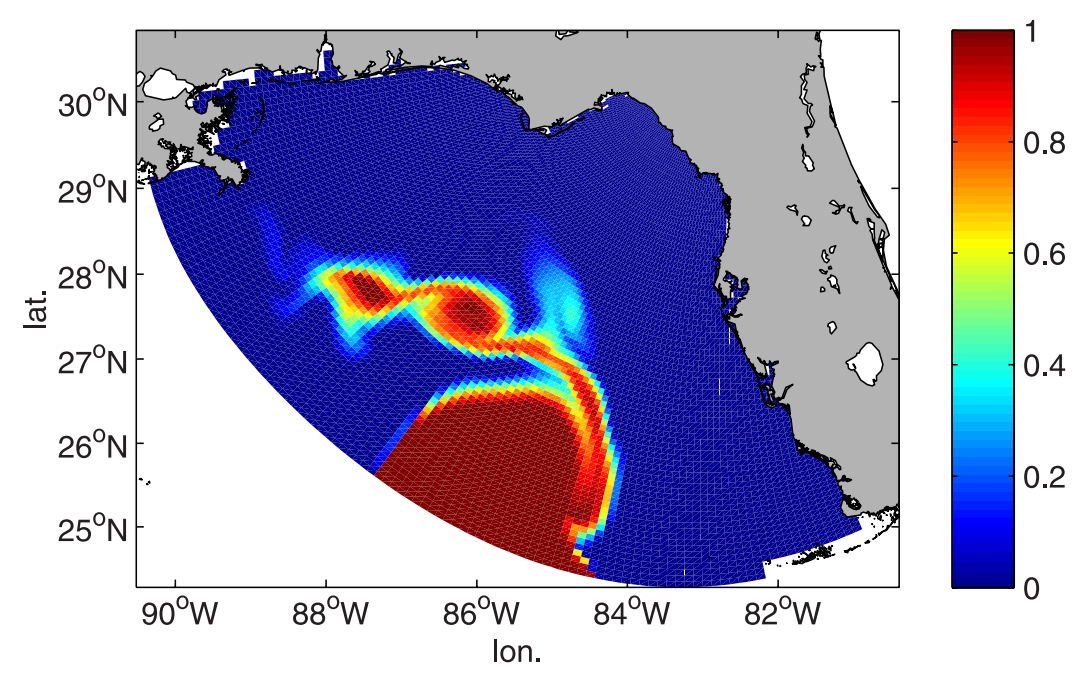

Figure 13. Tracer representing LC water on 22 March 2004. A tracer value of 0 represents no LC water, and 1 indicates only LC water. All values between 0 and 1 show the degree of mixing of LC water with the surrounding waters.

affected and are identical to the model output discussed in the model verification section. The addition of this tracer reveals a very rich and clear signal of the mesoscale flow generated by the LC. Figure 13 shows how water on the edge of the LC is peeled off from the LC through a growing perturbation generated upstream of the LC. This band of LC water begins to structure itself and breaks down in anticyclonic eddies. Such filament formation occurred 16 times per year; this process appears to be an active mechanism of detaching water from the LC.
[66] The integral of this tracer over a given volume represents the amount of $\mathrm{LC}$ water present in this volume. In order to quantify the amount of LC water reaching the shelf we integrated this tracer over the model domain with a depth shallower than $100 \mathrm{~m}$. It was necessary to integrate the WFS ROMS model for 2 years to reach a statistical equilibrium (Figure 14). At equilibrium, the WFS contains about $5.510^{11} \mathrm{~m}^{3}$ water of LC origin or $9 \%$ of the total shelf volume. Figure 15 shows the rate by which LC water reaches the shelf. Each peak in this figure shows the

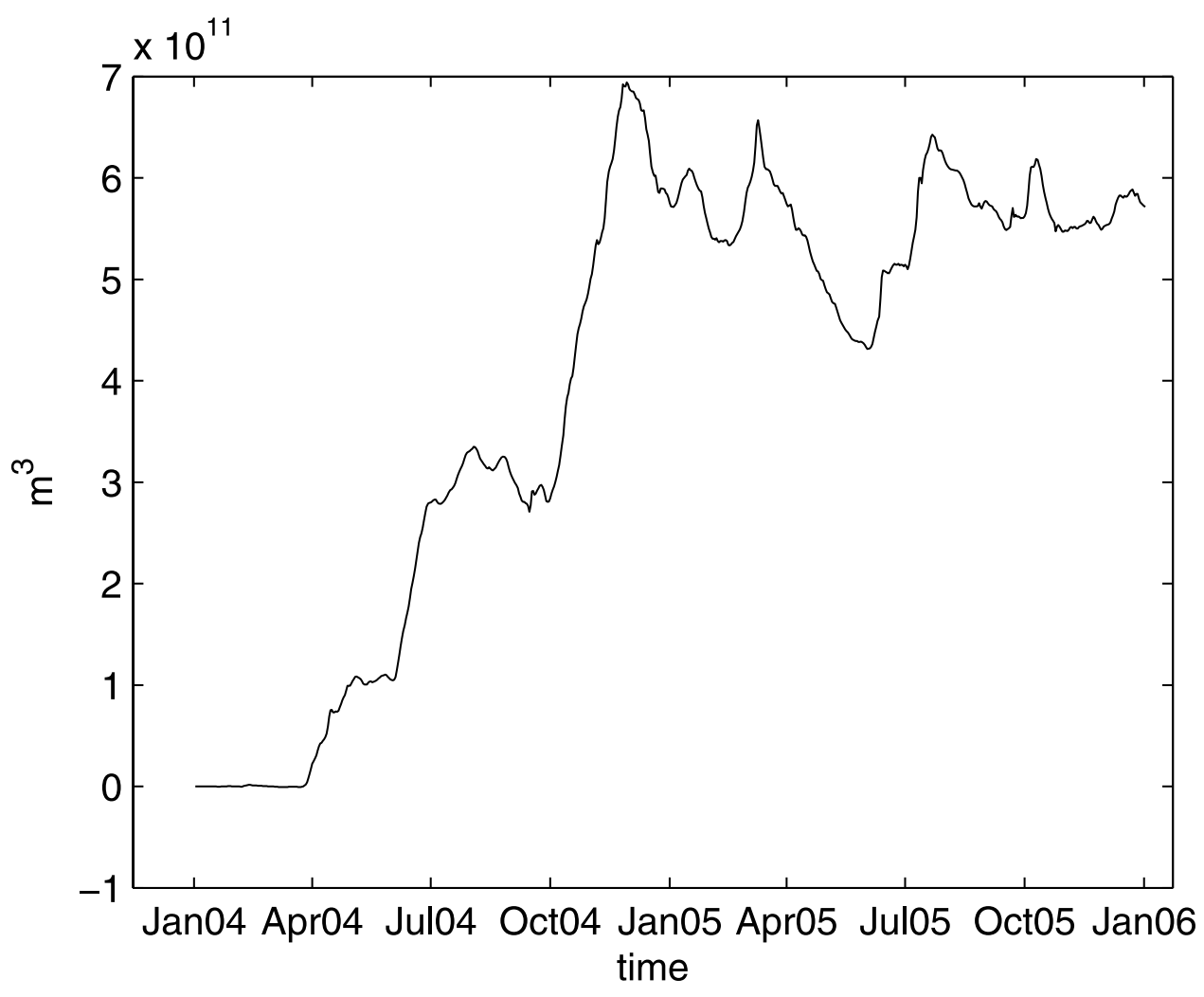

Figure 14. Change of LC water volume on the WFS shelf with time. 


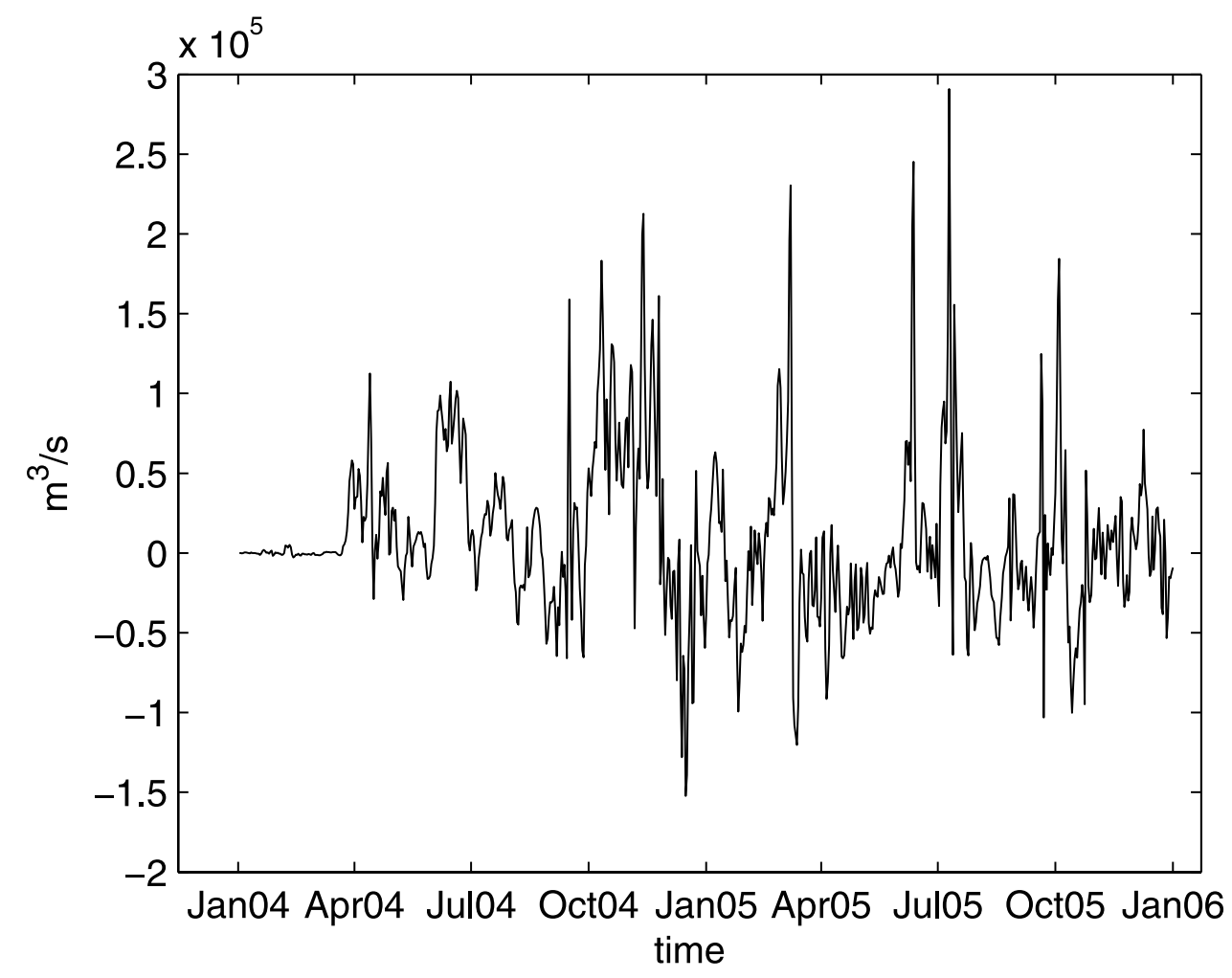

Figure 15. The rate of increase and decrease of LC water on the WFS shelf.

intrusion of LC water. The first peak corresponds to the filament shown in Figure 13 reaching the shelf. The flux of LC water associated to this event is about $510^{4} \mathrm{~m}^{3} \mathrm{~s}^{-1}$ and the total volume reaching the shelf is $10^{7} \mathrm{~m}^{3}$. The large increase between 6 October 2004 and 5 November 2004 of $2.510^{11} \mathrm{~m}^{3}$ corresponds to an anticyclonic LC vortex of about $80 \mathrm{~km}$ diameter and $70 \mathrm{~m}$ depth reaching the DeSoto Canyon. This eddy is dissipated by the contact with the bottom topography and its water is partially advected on the shelf. The total volume of this eddy, $3.510^{11} \mathrm{~m}^{3}$, computed from its diameter and depth, is in agreement with the total LC water increase on the shelf. Although such vortices are less frequent than filaments reaching the shelf, eddies transport more LC water than filaments and play an equally important role in transporting LC water onto the shelf. A large decrease of LC water on the shelf occurs on 15 December 2004 and at the end of March 2005: a large amount of LC water, after being advected southward by the shelf circulation, exits the shelf at the southeastern end of the WFS.

[67] Figure 3 shows a filament of LC waters as observed by the GOES satellite SST on 15 January 2004. At this moment of the year the LC water is several degrees warmer that its surrounding water. The structure and size of the filament agrees with the filament generated in the tracer experiment (Figure 13). Both filaments are still attached to the eastern side of the LC and they are about the break in smaller structures. However, the model is not able to reproduce the exact timing of these events. We attribute this to the fact that these structures are generated by instability processes and are therefore nondeterministic. Data assimilation is probably necessary to reproduce the correct timing of such nonlinear processes. However, com- parison with observations shows that (1) the overall structure of the filaments, (2) the size and propagation speed of LC generated vortices and (3) model currents at several in situ stations, match the observations reasonably well. This gives us confidence in the overall statistical properties of the LC water exchange obtained from this simulation. Despite the difficulties to validate directly these results, this kind of tracer study provides useful information to track LC water and to study LC generated mesoscale variability in a statistical sense.

\section{Conclusions}

[68] The present work describes a ROMS (Regional Ocean Modeling System) implementation of the West Florida Shelf (WFS) nested in the North Atlantic Hybrid Coordinate Model (NAT HYCOM). The model nesting allows to include a realistic Loop Current (LC) in the WFS model. The objective of the WFS ROMS is the study of the mesoscale flow generated by the LC. The present work was divided into three successive steps. First, the model was validated by comparing its results to various in situ measurements. This model was calibrated by a sensitivity analysis to ensure that the model configuration is appropriate. Finally, the model results were interpreted by examining the mesoscale variability created by the LC.

[69] In the model validation phase, we assessed the skill of the nested model relative to the outer model. For most locations, we obtained a positive skill meaning that the nested solution is closer to the observations than the outer model despite the fact that the model nesting boundary crosses a strong and variable current. However, the model skill in temperature is negative near the shelf break. This 
might be due to the fact that isopycnal coordinates can handle an abrupt bathymetry change better than terrainfollowing coordinates.

[70] A sensitivity study showed that a rather strong SST relaxation implemented as a heat flux correction [Barnier et al., 1995] is necessary to accurately reproduce the in situ temperature at the surface and at depth. Only under stratified conditions, the bottom temperature is independent of the SST relaxation.

[71] We showed also the benefit of the optimal interpolated winds on the shelf currents in a model nesting configuration. The use of this wind product corresponds to an average RMS error decrease of $10 \%$ on the shelf surface currents compared to using NOGAPS winds. The problems associated with the use of different wind forcings in the nested model and the outer model are outweighed by the improved accuracy on the shelf.

[72] K-Profile Parametrization and Mellor-Yamada 2.5 (MY) turbulence scheme gave quantitatively comparable results onto the shelf and are in reasonable agreement with the observations. Overall, the RMS error is however slightly lower with MY (but statistically significant). Increased vertical resolution on the shelf (32 levels compared to 16) improves the model results. But its relative improvement ( $5 \%$ in RMS error reduction) is small compared to the RMS error reduction obtained by relaxing the model to satellite SST, for example. This suggests that the main limitation of the shelf modeling are the atmospheric heat fluxes and not primarily the vertical resolution.

[73] The model experiments with higher resolution indicate that a finer model grid improves the model solution because it admits a more representative bathymetry. The high-resolution model using only an interpolated bathymetry (from the coarse grid model) did not ameliorate the model results. However, changes in the model resolution and bathymetry do not modify the statistics of the Loop Current sea surface height variability.

[74] The model nesting allowed us to study the propagation of a vortex generated by the LC. The speed and trajectory of this eddy were explained by the gradient of ambient potential vorticity between the $\mathrm{LC}$ and the shelf break. The theoretical propagation speed [Cushman-Roisin, 1994] based on the model density is in agreement with the model vortex propagation speed and the speed derived from altimetry.

[75] By introducing a tracer identifying LC water, we have been able to study the main processes for transporting LC water on the shelf, namely filaments and eddy detachment. The flux of LC water reaching the shelf has been estimated on the basis of this tracer experiment. The total amount of LC water on the shelf stabilized at $9 \%$ of the WFS volume (delimited by the $100-\mathrm{m}$ isobath). This equilibrium was reached after one year, suggesting that this is the order of magnitude of the residence time of $\mathrm{LC}$ water on the shelf. The size and structure of the filaments in the model agrees with filaments observed in satellite SST. However, the model is unable to reproduce the exact timing of these filaments generation. Data assimilation is probably necessary to constrain the generation of these instability processes and will be addressed in future work.

[76] The comparison with ADCP data showed also that the nested model can also give worse result than the outer model near the open boundary. The analysis of the model data revealed that the model nesting create sometimes spurious upwelling at the boundary. A perfect match of the bathymetry is difficult (if not impossible) to achieve within the flow relaxation zone if the models have a different vertical coordinate system. A slight mismatch can create an unrealistic vertical velocity and thus a spurious upwelling or downwelling in the flow relaxation zone. A nesting scheme which does not alter the divergence of the horizontal flow even under a slightly different bathymetry would reduce such problems at the nesting boundary and facilitate the nesting of models with different vertical coordinate systems.

[77] Some general conclusions independent from the particular model and site can also be drawn on the basis of our results. The present study shows that a resolution of about $6 \mathrm{~km}$ is sufficient to reproduce generation of filaments and eddies produced by instabilities from a strong baroclinic current such as the LC. The study also demonstrates that the subsequent propagation of the vortex can be adequately modeled provided that the background stratification is realistic. In the present experiments, it even turned out that the background stratification is more important than the background velocity to simulate the vortex propagation.

[78] Acknowledgments. This work was supported by the SouthEast U.S. Atlantic Coastal Ocean Observing System (SEACOOS) program and the Global Ocean Prediction with the Hybrid Coordinate Ocean Model (HYCOM) program of the US Office of Naval Research (grants N0001402-1-0972 and N00014-04-1-0676, respectively). In situ data are attributed to the Ocean Circulation Group. Particular thanks go to Rick Cole, Jeff Donovan, Jay Law, Dennis Mayer, Cliff Merz, Jyotika Virmani, and Vembu Subramanian. Ole Martin Smedstad is acknowledged for his help with the HYCOM results. The sea level anomalies have been produced by the CLS Space Oceanography Division, Toulouse, France. The F.R.S.-FNRS, Belgium, is also acknowledged for the funding of the current postdoctoral positions of A. Barth and A. Alvera-Azcárate.

\section{References}

Barnier, B., L. Siefridt, and P. Marchesiello (1995), Thermal forcing for global ocean circulation model using a 3-year climatology of ECMWF analyses, J. Mar. Syst., 6, 363-380.

Barron, C. N., A. B. Kara, P. J. Martin, R. C. Rhodes, and L. F. Smedstad (2006), Formulation, implementation and examination of vertical coordinate choices in the Global Navy Coastal Ocean Model (NCOM), Ocean Modell., 11, 347-375.

Barth, A., A. Alvera-Azcárate, and R. H. Weisberg (2007), Benefit of nesting a regional model into a large-scale ocean model instead of climatology. Application to the West Florida Shelf, Cont. Shelf Res., 28, $561-573$.

Bertino, L., M. S. George, and K. A. Lisæter (2007), Validation of a Hybrid Coordinate Ocean Model for the Indian Ocean, paper presented at Layered Ocean Model Workshop, Rosenstiel School of Mar. and Atmos. Sci., Bergen, Norway.

Browning, G. L., W. R. Holland, H.-O. Kreiss, and S. J. Worley (1990), An accurate hyperbolic system for approximately hydrostatic and incompressible oceanographic flows, Dyn. Atmos. Oceans, 14, 303-332.

Chapman, D. (1985), Numerical treatment of cross-shelf open boundaries in a barotropic coastal ocean model, J. Phys. Oceanogr., 15, 1060-1075.

Chassignet, E. P., H. E. Hurlburt, O. M. Smedstad, G. R. Halliwell, P. J. Hogan, A. J. Wallcraft, R. Baraille, and R. Bleck (2007), The HYCOM (HYbrid Coordinate Ocean Model) Data Assimilative System, J. Mar. Syst., 65, 60-83.

Cooper, M., and K. Haines (1996), Data assimilation with water property conservation, J. Geophys. Res., 101(C1), 1059-1077.

Cushman-Roisin, B. (1994), Introduction to Geophysical Fluid Dynamics, 320 pp. Prentice Hall, Englewood Cliffs, N. J.

Demirov, E., N. Pinardi, C. Fratianni, M. Tonani, L. Giacomelli, and P. Mey (2003), Assimilation scheme of the Mediterranean Forecasting System: Operational implementation, Ann. Geophys., 21, 189-204.

DeRada, S., J. C. Kindle, S. Anderson, I. Shulman, B. Penta, A. Wallcraft, and P. Martin (2006), Nesting regional models in a generalized coordinate 
(hybrid) global ocean Model, Eos Trans. American Geophysical Union, 87(36), Ocean Sci. Meet. Suppl., Abstract OS26O-22.

Di Lorenzo, E., A. J. Miller, N. Schneider, and J. C. McWilliams (2005), The warming of the California Current: Dynamics and ecosystem implications, J. Phys. Oceanogr., 35, 336-362.

Dorandeu, J., and P. Y. Le Traon (1999), Effects of global mean atmospheric pressure variations on mean sea level changes from TOPEX Poseidon, J. Atmos. Oceanic Technol., 16, 1279-1283.

Durski, S., S. Glenn, and D. Haidvogel (2004), Vertical mixing schemes in the coastal ocean: Comparison of the level 2.5 Mellor-Yamada scheme with an enhanced version of the K profile parameterization, J. Geophys. Res., 109, C01015, doi:10.1029/2002JC001702.

Fairall, C., E. Bradley, D. Rogers, J. Edson, and G. Young (1996), Bulk parameterization of air-sea fluxes for TOGA COARE, J. Geophys. Res. 101, 3747-3764.

Flather, R. (1976), A tidal model of the northwest European continental shelf, Mem. Soc. R. Sci. Liege, 6(10), 141-164.

Halliwell, G. R. (2004), Evaluation of vertical coordinate and vertical mixing algorithms in the HYbrid-Coordinate Ocean Model (HYCOM), Ocean Modell., 7, 285-322, doi:10.1016/j.ocemod.2003.10.002.

Hamilton, P., and T. N. Lee (2005), Eddies and jets over the slope of the northeast Gulf of Mexico, in Circulation in the Gulf of Mexico: Observations and Models, Geophys. Monogr. Ser., vol. 161, edited by W. Sturges and A. Lugo-Fernandez, pp. 123-142, AGU, Washington, D. C.

He, R., and R. H. Weisberg (2002), West Florida shelf circulation and temperature budget for the 1999 spring transition, Cont. Shelf Res., 22 , $719-748$

He, R., and R. H. Weisberg (2003a), West Florida Shelf circulation and temperature budget for the 1998 fall transition, Cont. Shelf Res., 23, $777-800$

He, R., and R. H. Weisberg (2003b), A loop intrusion case study on the West Florida Shelf, J. Phys. Oceanogr., 33, 465-477.

He, R., R. H. Weisberg, H. Zhang, F. Muller-Karger, and R. W. Helber (2003), A cloud-free, satellite-derived, sea surface temperature analysis for the West Florida Shelf, Geophys. Res. Lett., 30(15), 1811, doi:10.1029/2003GL017673.

He, R., Y. Liu, and R. H. Weisberg (2004), Coastal ocean wind fields gauged against the performance of an ocean circulation model, Geophys. Res. Lett., 31, L14303, doi:10.1029/2003GL019261.

Hetland, R. D., Y. Hsueh, R. R. Leben, and P. P. Niiler (1999), A loop current-induced jet along the edge of the West Florida Shelf, Geophys. Res. Lett., 26, 2239-2242.

Hu, C., J. R. Nelson, E. Johns, Z. Chen, R. H. Weisberg, and F. E. MüllerKarger (2005), Mississippi River water in the Florida Straits and in the Gulf Stream off Georgia in summer 2004, Geophys. Res. Lett., 32 L14606, doi:10.1029/2005GL022942.

Kara, A. B., and H. E. Hurlburt (2006), Daily inter-annual simulations of SST and MLD using atmospherically forced OGCMs: Model evaluation in comparison to buoy time series, J. Mar. Syst., 62, 95-119.

Kara, A. B., A. J. Wallcraft, P. J. Martin, and E. P. Chassignet (2008), Performance of mixed layer models in simulating SST in the equatorial Pacific Ocean, J. Geophys. Res., 113, C02020, doi:10.1029/2007JC004250.

Kourafalou, V. H., R. S. Balotro, T. M. Chin, A. Srinivasan, A. J. Wallcraft, C. B. Paris, T. N. Lee, P. B. Ortner, and E. M. Johns (2006), Interdisciplinary nested simulations with the South Florida Regional SoFLAHYCOM Model, Eos Trans. American Geophysical Union, 87(36), Ocean Sci. Meet. Suppl., Abstract OS26N-23.

Kundu, P. K., and J. S. Allen (1976), Some three-dimensional characteristics of low-frequency current flucutuations near the Oregon coast., $J$. of Phys. Oceanogr., 6, 181-199.

Large, W., J. McWilliams, and S. Doney (1994), Oceanic vertical mixing: A review and a model with a nonlocal boundary layer parameterization, Rev. Geophys., 32, 363-403.

Leben, R. R. (2005), Altimeter-derived loop current metrics, in Circulation in the Gulf of Mexico: Observations and Models, Geophys. Monogr. Ser., vol. 161, edited by W. Sturges and A. Lugo-Fernandez, pp. 181-201, AGU, Washington, D. C.
Le Traon, P.-Y., F. Nadal, and N. Ducet (1998), An improved mapping method of multisatellite altimeter data, J. Atmos. Oceanic Technol., 15, $522-534$

Li, Z., and R. Weisberg (1999), West Florida Shelf response to upwelling favorable wind forcing: Kinematics, J. Geophys. Res., 104, 13,50713,527

Liu, Y., and R. Weisberg (2007), Ocean currents and sea surface heights estimated across the West Florida Shelf, J. Phys. Oceanogr., 37, 16971713.

Marchesiello, P., J. McWilliams, and A. Shchepetkin (2001), Open boundary condition for long-term integration of regional oceanic models, Ocean Modell., 3, 1-20.

Mellor, G., and T. Yamada (1982), Development of a turbulence closure model for geophysical fluid problems, Rev. Geophys., 20, 851-875.

Murphy, A. H. (1988), Skill scores based on the mean square error and their relationships to the correlation coefficient, Mon. Weather Rev, 116 , $2417-2424$

Oliger, J., and A. Sundstrom (1978), Theoretical and practical aspects of some initial and boundary value problems in fluid dynamics, SIAM J. Appl. Math., 35(3), 419-446.

Shchepetkin, A., and J. McWilliams (2003), A method for computing horizontal pressure-gradient force in an oceanic model with a nonaligned vertical coordinate, J. Geophys. Res., 108(C3), 3090, doi:10.1029/ 2001JC001047.

Shchepetkin, A., and J. McWilliams (2005), The Regional Oceanic Modeling System: A split-explicit, free-surface, topography-following-coordinate ocean model, Ocean Modell., 9, 347-404.

Smedstad, O., J. A. Cummings, E. J. Metzger, P. J. Hogan, H. E. Hurlburt A. J. Wallcraft, and E. P. Chassignet (2007), Real-time HYCOM nowcast/ forecast systems, paper presented at 11th HYCOM Consortium Meeting, Nav. Res. Lab., Stennis Space Center, Miss.

Song, Y. T., and D. Haidvogel (1994), A Semi-implicit ocean circullation model using a generalized topography following coordinate system, J. Comput. Phys., 115, 228-248.

Spall, M. A., and W. R. Holland (1991), A nested primitive equation model of oceanic application, J. Phys. Oceanogr., 21, 205-220.

Srinivasan, A., Z. Garraffo, and M. Iskandarani (2007), Abyssal Circulation in the Indian Ocean from a high resolution HYCOM, paper presented at Layered Ocean Model Workshop,Rosenstiel School of Mar. and Atmos, Sci., Bergen, Norway.

Tester, P. A., and K. A. Steidinger (1997), Gymnodinium breve red tide blooms: Initiation, transport, and consequences of surface circulation, Limnol. Oceanogr., 42, 1039-1051.

Walsh, J., et al. (2003), Phytoplankton response to intrusions of slope water on the West Florida Shelf: Models and observations, J. Geophys. Res., 108(C6), 3190, doi:10.1029/2002JC001406.

Weisberg, R. H., and R. He (2003), Local deep-ocean forcing contributions to anomalous water properties of the West Florida Shelf, J. Geophys. Res., 108(C6), 3184, doi:10.1029/2002JC001407.

Weisberg, R. H., B. D. Black, and Z. Li (2000), An upwelling case study on Florida's west coast, J. Geophys. Res., 105, 11,459-11,469.

Weisberg, R. H., L. Zhenjiang, and F. Muller-Karger (2001), West Florida shelf response to local wind forcing: April 1998, J. Geophys. Res., 106, $31,239-31,262$

Weisberg, R. H., R. He, Y. Liu, and J. I. Virmani (2005), West Florida Shelf circulation on synoptic, seasonal, and interannual time scales, in Circulation in the Gulf of Mexico: Observations and Models, Geophys. Monogr Ser., vol. 161, edited by W. Sturges and A. Lugo-Fernandez, pp. $325-$ 347, AGU, Washington, D. C.

Wilkin, J. L., H. G. Arango, D. B. Haidvogel, C. S. Lichtenwalner, S. M. Glenn, and K. S. Hedström (2005), A regional ocean modeling system for the Long-term Ecosystem Observatory, J. Geophys. Res., 110, C06S91, doi:10.1029/2003JC002218.

A. Alvera-Azcárate and R. H. Weisberg, College of Marine Science, University of South Florida, St. Petersburg, FL 33701, USA

A. Barth, Department of Astrophysics Geophysics and Oceanography, University of Liege, Liege B-4000, Belgium. (a.barth@ulg.ac.be) 